\title{
Distinct diagnostic and prognostic values of $\gamma$-aminobutyric acid type A receptor family genes in patients with colon adenocarcinoma
}

\author{
LING YAN $^{1}$, YI-ZHEN GONG ${ }^{1}$, MENG-NAN SHAO $^{2}$, GUO-TIAN RUAN $^{1}$, HAI-LUN XIE $^{1}$, XI-WEN LIAO $^{3}$, \\ XIANG-KUN WANG ${ }^{3}$, QUAN-FA HAN ${ }^{3}$, XIN ZHOU $^{3}$, LI-CHENG ZHU ${ }^{4}$, FENG GAO ${ }^{1}$ and JIA-LIANG GAN ${ }^{1}$ \\ ${ }^{1}$ Department of Colorectal and Anal Surgery, The First Affiliated Hospital of Guangxi Medical University; \\ ${ }^{2}$ Life Sciences Institute, Guangxi Medical University; ${ }^{3}$ Department of Hepatobiliary Surgery, The First Affiliated \\ Hospital of Guangxi Medical University; ${ }^{4}$ Department of Immunology, School of Preclinical Medicine, \\ Guangxi Medical University, Nanning, Guangxi Zhuang Autonomous Region 530021, P.R. China
}

Received July 11, 2019; Accepted February 7, 2020

DOI: 10.3892/ol.2020.11573

\begin{abstract}
In the present study, the significance of $G A B A_{A}$ genes in colon adenocarcinoma (COAD) were investigated from the view of diagnosis and prognosis. All data were achieved from The Cancer Genome Atlas. Overall survival was analyzed by the Kaplan-Meier analyses and Cox regression model and the hazard ratios and $95 \%$ confidence interval were calculated for computation. The Database for Annotation, Visualization and Integrated Discovery, and the Biological Networks Gene Ontology (BiNGO) softwares were applied to assess the biological processes and Kyoto Encyclopedia of Genes and Genomes (KEGG) was used for pathway analysis to predict the biological function of $G A B A_{A}$ genes. The associated Gene Ontology and KEGG pathways were conducted by Gene Set Enrichment Analysis (GSEA). From receiver operating characteristics curves analysis, it was found that the expression of $G A B R, \gamma$-aminobutyric acid type A receptor $G A B R A 2, G A B R A 3, G A B R B 2, G A B R B 3$, $G A B R G 2, G A B R G 3, G A B R D, G A B R E$ were correlated with COAD occurrence $[\mathrm{P}<0.0001$, area under the curve (AUC) $>0.7]$. The low expression of the $G A B R B 1, G A B R D$, $G A B R P$ and $G A B R Q$ in genes after tumor staging adjustment were positively correlated with the overall survival rate $[\mathrm{P}=0.049$, hazard ratio $(\mathrm{HR})=1.517,95 \%$ confidence interval $(\mathrm{CI})=1.001-2.297 ; \mathrm{P}=0.006, \mathrm{HR}=1.807,95 \% \mathrm{CI}=1.180-2.765$; $\mathrm{P}=0.005, \mathrm{HR}=1.833,95 \% \mathrm{CI}=1.196-2.810 ; \mathrm{P}=0.034$, $\mathrm{HR}=1.578,95 \% \mathrm{CI}=1.036-2.405)$. GSEA showed enrichment
\end{abstract}

Correspondence to: Professor Jia-Liang Gan, Department of Colorectal and Anal Surgery, The First Affiliated Hospital of Guangxi Medical University, 6 Shuang Yong Road, Nanning, Guangxi Zhuang Autonomous Region 530021, P.R. China

E-mail: gj15172@163.com

Key words: $\gamma$-aminobutyric acid type A receptor, mRNA, colon adenocarcinoma, diagnosis, prognosis of cell matrix adhesion, integrin binding, angiogenesis, endothelial growth factor and endothelial migration regulation in patients with COAD with $G A B R D$ overexpression. $G A B R B 1$, $G A B R D, G A B R P$ and $G A B R Q$ were associated with the prognostic factors of COAD. The expression levels of GABRA2, GABRA3, GABRB2, GABRB3, GABRG2, GABRD and $G A B R E$ may allow differentiation between tumor tissues and adjacent normal tissues.

\section{Introduction}

Colorectal cancer (CRC) is a type of malignant tumor originated from colon and rectum epithelium (1). Most cases of CRC develop slowly through normal mucosal adenoma-cancer sequence for several years and it is one of the most common malignant tumors in the clinic worldwide $(2,3)$. In 2018, the global incidence of colorectal cancer was third from the top among the 36 types of cancer and the mortality rate ranked second and 1.8 million individuals were diagnosed with colorectal cancer in the world (4), the number of deaths due to colorectal cancer was approximately 881,000 . Colon cancer is a type of colorectal cancer and accounts for a large proportion of colorectal cancer cases approximately $60.9 \%$ in the world in $2018(4,5)$. The primary risk factors associated with the disease are elderly, male sex, increased levels of fat consumption, high level of red meat and processed food consumption, lack of exercise, smoking, high alcohol intake ( $>1$ drink/day) (6), obesity and being tall $(4,7)$. The treatment methods of COAD included radiotherapy, surgery, targeted therapy and chemotherapy. Although a great deal of effort has been made to understand the underlying molecular mechanisms of the occurrence and development of COAD, the prevention and treatment of early-onset COAD is still a challenge for researchers (8). Therefore, sensitive and specific biomarkers are needed to improve early diagnosis, aid the management of individualized therapy and predict the prognosis of patients at different stages of the COAD.

$\gamma$-Aminobutyric acid $(G A B A)$ is the principal inhibitory neurotransmitter in the mammalian brain. $\gamma$-Aminobutyric 
acid type $\mathrm{A}\left(G A B A_{A}\right)$ receptors are the primary mediators of inhibitory neurotransmission in the mature brain, which also functions as an agonist-gated ion channel that mediates rapid synaptic inhibition in the mammalian central nervous system (9). The $G A B A_{A}$ receptor subunit is mainly expressed in the cerebellum and its receptor is located in cerebellum, but $G A B A_{A}$ is also expressed in testis and CD4-T-cells $(10,11)$. The $G A B A_{A}$ receptor $(G A B R)$ subunits are a superfamily consisting of 19 subunits: $\alpha 1-\alpha 6$ (GABRA1, GABRA2, GABRA3, GABRA4, GABRA5 and GABRA6); $\beta 1-\beta 3$ (GABRB1, $G A B R B 2$ and GABRB3); $\gamma 1-\gamma 3(G A B R G 1, G A B R G 2$ and GABRG3); $\delta$ (GABRD); $\varepsilon$ (GABRE); $\pi(G A B R P) ; \theta(G A B R Q)$; and $\rho 1-\rho 3$ (GABRR1, GABRR2, GABRR3) $(9,12,13)$. However, the data regarding the mRNA expression levels of five GABAA family genes, including GABRAl, GABRA5, GABRG1, GABRA6 and GABRR3, were not available in The Cancer Genome Atlas (TCGA) database. Thus, only 14 genes were analyzed in the present study. Previous study showed that overexpressed $G A B R D$ was observed in $89 \%$ of cases and had a weak negative correlation with tumor proliferation, proliferative-independent genes are upregulated in tumors and $G A B A_{A}$ receptors might play a role in the differentiation of tumor cells (14). However, the diagnostic and prognostic value of GABRD and its family members had not been thoroughly and systematically described. In the present study, the role of the GABA family in colon cancer was studied using the TCGA database to obtain survival-associated and $\mathrm{GABA}_{A}$ family expression in patients with COAD patients and the diagnostic and prognosis value of the mRNA expression levels of $\mathrm{GABA}_{A}$ family genes were investigated. A few online data portals were applied to analyze functions and signaling pathways to predict the function of these genes.

\section{Materials and methods}

Data preparation. The mRNA expression levels and clinical information associated with COAD, including sex, age and tumor-non-metastasis (TNM) stage (8), were obtained from TCGA (cancer.gov/tcga). Overall, 456 patients were performed by mRNA sequencing. The expression data included 480 tumor tissues and 41 adjacent normal tissues. The Bioconductor package (edgeR, version 3.24.3; R, version 3.6.0 software; rstudio, version 1.2.5019) was used to standardize and correct the original data (15). Genes with P-value $<0.05$ and $\log _{2}$ fold-change $(\mathrm{FC}) \mid>2$ were deemed to be significantly different. These genes were regarded as differentially expressed genes (DEGs) (16). First, tumor tissues and adjacent normal tissues data were isolated and then the gene expression data were integrated with clinical information. Finally, patients who had repetition of the data, a survival time of 0 days or no follow-up data were excluded. In the end, 438 tumor tissues and 41 adjacent normal tissues were analyzed in the final research.

mRNA co-expression and functional analysis. In order to analyze the biological pathways and significance of the $G A B A_{A}$ family genes, a set of functional enrichment analyses were carried out using Database for Annotation, Visualization and Integrated Discovery (DAVID 6.8, david.ncifcrf. gov/home.jsp) (17,18). Enriched P-values $<0.05$ had statistical significance. These included the terms Gene Ontology (GO) functional examination and the Kyoto Encyclopedia of Genes and Genomes (KEGG) pathway analysis. The functional detection of Molecular functional (MF), cell component (CC) and Biological process (BP) were based on the analysis of $\mathrm{GO}$ terminology.

Biological Networks Gene Ontology (BiNGO) (19) was chosen as a tool for GO functional analysis. BiNGO predicted gene function through the consequences of correlation analysis. Gene Multiple Association Network Integration Algorithm (GeneMANIA) was applied for the calculation of the 14 genes of $G A B A_{A}$ family $(20,21)$. The Search Tool for the Retrieval of Interacting Genes/Proteins (STRING) database was used to evaluate protein-protein interactions (22) and was applied to evaluate the function and physiological relationships between the $G A B A_{A}$ family genes. A total score $>0.15$ was considered to be statistically significant.

Co-representation matrix of $G A B A_{A}$ families. The correlation between $G A B A_{A}$ family genes in COAD was determined using Pearson correlation coefficient analysis. An absolute value of correlation coefficient $>0.4$ was considered strong correlation.

Gene expression level characteristics. Metabolic Gene Rapid Visualizer (MERAV) was performed to create boxplots of the differentially expressed genes of the $G A B A_{A}$ family in primary colon cancer tissue and normal colon tissue (23). $G A B A_{A}$ gene expression levels in tumor and adjacent normal tissues were used to construct vertical scatterplots. In addition, the differential expressed genes of the $G A B A_{A}$ family were screened with the median cut-off values of all genes. Patients who possessed higher value than the median values of $G A B A_{A}$ genes expression were classified as the high expression group and the other patients were classified into the low expression group.

Diagnostic forecast. GraphPad Prism version 7 (GraphPad Software) was used to construct receiver operating characteristics (ROC) curves to investigate the prognostic value of the $G A B A_{A}$ genes in patients with COAD in TCGA database. Then the correlation between diagnosis associated genes and tumor stage was investigated using a Spearman's test and Gene Expression Profiling Interactive Analysis (24). The normalized diagnostic value of $\mathrm{P}<0.05$ was considered to indicate a statistically significant difference.

Survival analysis. According to the median cut-off value of each $G A B A_{A}$ genes, the patients were categorized into low and high expression groups. P-value and overall survival (OS) of the $G A B A_{A}$ gene family and clinical data were calculated using Kaplan-Meier analysis and a log-rank test.

To assess the prognostic model thoroughly, a Cox proportional risk regression model for univariate and multivariate survival tests was performed. After adjusting the clinical characteristics, 95\% confidence intervals (CIs) and hazard ratios (HRs) were calculated by conducting Cox proportional risk regression model.

Joint-effects analysis. Based on previous survival analysis, joint-effects analysis $(25,26)$ of the prognostic associated genes (GABRB1, GABRD, GABRP and GABRQ) was performed to 
analyze the effect of polygenes on the survival of patients. Use the following combinations for joint analysis:1) GABRB1 and $G A B R D$; 2) GABRB1 and GABRP; 3) GABRB1 and GABRQ; 4) $G A B R D$ and $G A B R P$; 5) GABRD and $G A B R Q$; 6) GABRP and $G A B R Q$; 7) GABRB1, GABRD and GABRP; 8) GABRB1, $G A B R D$ and GABRQ; 9) GABRB1, GABRP and GABRQ; 10) $G A B R D, G A B R P$ and $G A B R Q$. Each combination was divided groups based on the median gene expression mentioned earlier (e.g. combination A and B: Group 1=low A+ low B, group $2=$ low $\mathrm{A}+$ high $\mathrm{B}$ or high $\mathrm{A}+$ low $\mathrm{B}$, group $3=$ high $\mathrm{A}+$ high $\mathrm{B}$; combination $\mathrm{A}, \mathrm{B}$ and $\mathrm{C}$ : Group $1=$ low $\mathrm{A}+$ low $\mathrm{B}+$ low $\mathrm{C}$, group $2=$ low $\mathrm{A}+$ low $\mathrm{B}+$ high $\mathrm{C}$ or low $\mathrm{A}+$ high $\mathrm{B}+$ low $\mathrm{C}$ or high $\mathrm{A}+$ low $\mathrm{B}+$ low $\mathrm{C}$, group $3=$ high $\mathrm{A}+$ high $\mathrm{B}+$ low $\mathrm{C}$ or high $\mathrm{A}+$ low $\mathrm{B}+$ high $\mathrm{C}$ or low $\mathrm{A}+$ high $\mathrm{B}+$ high $\mathrm{C}$; group $4=$ high $\mathrm{A}+$ high $\mathrm{B}+$ high $\mathrm{C}$ ). According to the above combination, the Cox proportional risk regression model was adjusted for statistical significance factors (i.e., TNM stage). Kaplan-Meier method and log-rank test were used to evaluate the prognostic value of $G A B A_{A}$ genes combination expression in each group.

Nomogram. A nomogram was used to assess the association between $G A B R B 1, G A B R D, G A B R P, G A B R Q$ and medical rank (gender, age, stage) in terms of OS for patients with COAD. In addition, the potential of these four genes in predicting clinical grade was evaluated.

In terms of clinical data and survival analysis, only tumor stage and $G A B R B 1, G A B R D, G A B R P$ and $G A B R Q$ expression level entered the risk model after being adjusted by cox proportional hazard regression model. The risk score for all factors were calculated as well as the 1-, 2-, 3-, 4- and 5-year survival rates (27).

Gene set enrichment analysis (GSEA). In order to explore the differences in pathway and biological functions between low- and high-expression groups of the prognostic $G A B A_{A}$ genes, the expression profile of the full-genome dataset in TCGA group was divided into two groups according to the median prognostic $G A B A_{A}$ gene value. GSEA version 3.0 (software.broadinstitute.org/gsea/index.jsp) was applied to explore potential KEGG pathway and GO analysis within the Molecular Signatures Database of c2 curated gene set and c5 GO gene set (28). Criteria for significant enrichment gene sets in GSEA were: $\mathrm{P}<0.05$, False discovery rate $<0.25$.

Statistical analysis. Statistical analyses were performed using SPSS 20.0 (IBM Corp.) and R version 3.6.0 software. $\mathrm{P}<0.05$ was considered to indicate a statistically significant difference. DAVID was applied to analyze GO and KEGG pathways. The interactive network of the target genes was constructed using Cytoscape version 3.6.1. An unpaired t-test was used to compare data between COAD tumors and adjacent normal tissues. A Spearman's test was performed for the correlation analyses between TNM stages and GABRD expression levels.

\section{Results}

Gene expression dataset. Detailed baseline characteristics of 438 patients with COAD patients from TCGA database are summarized in Table I. Sex and age were not associated with
OS (all P>0.05), whereas TNM stage was significantly associated with OS (adjusted log-rank test $\mathrm{P}<0.001$ ).

Bioinformatics analysis of $G A B A_{A}$ family genes. The biological functional of the $G A B A_{A}$ genes was investigated using DAVID to evaluate GO functions and KEGG pathways (Fig. 1), BiNGO was applied to examine the enrichment outcomes (Fig. 2A) and the co-expression of the protein level was examined as shown in Fig. 2B. The interaction between $G A B A_{A}$ gene expression levels was presented in Fig. 3. The above results indicate that $G A B A_{A}$ genes were involved in the transport of substances and the formation of plasma membrane. In addition, the genes are strongly co-expressed and have complex networks of gene-gene and protein-protein interactions.

Through Pearson correlation coefficient analysis, it was found that there was a correlation between the expression levels of a single $G A B A_{A}$ gene. The expression level of $G A B R B 1$ was correlated with $G A B R A 2$ and $G A B R A 4$; GABRA4 were correlated with $G A B R B 1$; GABRA3 was correlated with GABRG3; GABRG3 were correlated with GABRA3, GABRQ and $G A B R G 2 ; G A B R Q$ were correlated with $G A B R G 3$ and $G A B R G 2$ (correlation coefficient $>0.4$; Fig. $4 \mathrm{~A}$ ).

Gene expression and diagnostic value of the $G A B A_{A}$ gene family. The vertical scattering map of $G A B A_{A}$ gene expression levels was shown in Fig. 4B, it showed that the results showed that GABRA2, GABRB2, GABRB3 and GABRG2 had low expression in tumor tissues; GABRB1, GABRD, GABRE and $G A B R P$ had high expression in tumor tissues. The correlation between gene expression and TNM stage showed that the expression levels of $G A B R D$ was significantly different in the four tumor stages (I, II, III and IV) from GEPIA (Fig. 4C). In our TCGA database, GABRD expression levels were associated with TNM stage also showed significantly weak positive correlation (Correlation Coefficient $=0.174$, Table II). The results of MERAV showed that the expression levels of GABRA2, GABRA3, GABRB2, GABRB3, GABRG3 and $G A B R R l$ in primary colon tumor tissues were lower compared with normal tissue (Fig. 5A, B, E, F, H and M), whereas the expression levels of GABRA4, GABRB1, GABRG2, GEBRD, $G A B R E, G A B R P$ and $G A B R R 2$ in primary colon tumor tissues was higher compared with normal colon tissue (Fig. 5C, D, G, I-L and N). In addition, ROC curves of the predicted expression levels of the $G A B A_{A}$ family genes in tumors and paired colon tissues was constructed (Fig. 6). The expression levels of GABRA2 (Fig. 6A), GABRA3 (Fig. 6B), GABRB2 (Fig. 6E), GABRB3 (Fig. 6F), GABRG2 (Fig. 6G), GABRG3 (Fig. 6H), GABRD (Fig. 6I) and GABRE (Fig. 6J) were significantly associated with the carcinogenesis of colon tumors (AUC >0.7).

Survival analysis. Univariate survival analysis demonstrated that tumor staging was the only factor associated with OS $\left(\mathrm{P}<0.001\right.$, Table I). The Kaplan-Meier curve of the $G A B A_{A}$ family genes were presented in Fig. 7A-N. Tumor staging was investigated using Cox proportional hazards regression model for multivariate survival tests, wherein the lower expression levels of $G A B R B 1, G A B R D, G A B R P$ and $G A B R Q$ were significantly correlated with favorable OS results (adjusted $\mathrm{P}=0.049, \mathrm{HR}=1.517,95 \% \mathrm{CI}=1.001-2.297$; adjusted $\mathrm{P}=0.006$, 
Table I. Demographic and clinical data for 438 patients with colon adenocarcinoma.

\begin{tabular}{|c|c|c|c|c|c|}
\hline Variables & Patients, $\mathrm{n}$ & No. of events ${ }^{a}$ & MST (days) & $\mathrm{HR}(95 \% \mathrm{CI})$ & Log-rank P-value ${ }^{b}$ \\
\hline Sex & & & & & 0.545 \\
\hline Male & 234 & 54 & 2,475 & 1 & \\
\hline Female & 204 & 44 & NA & $1.131(0.759-1.686)$ & \\
\hline $\operatorname{Age}^{c}$ (years) & & & & & 0.114 \\
\hline$\geq 65$ & 168 & 29 & 2,475 & 1 & \\
\hline$<65$ & 268 & 116 & NA & $1.420(0.919-2.194)$ & \\
\hline Tumor stage & & & & & $<0.001^{\mathrm{d}}$ \\
\hline IV & 61 & 31 & 858 & 1 & \\
\hline I & 73 & 4 & NA & $0.089(0.031-0.251)$ & \\
\hline II & 167 & 27 & 2,821 & $0.198(0.118-0.335)$ & \\
\hline III & 126 & 31 & NA & $0.360(0.218-0.596)$ & \\
\hline
\end{tabular}

${ }^{\mathrm{a}}$ Number of final events; ${ }^{\mathrm{b}}$ Adjusted for tumor stage. ${ }^{\mathrm{C}}$ Information of age was unknown in 2 patients. ${ }^{\mathrm{d}}$ Information of Tumor-Node-Metastasis stage was not reported in 11 patients; MST, median survival time; CI, confidence interval; HR, hazards ratio; NA, not available.

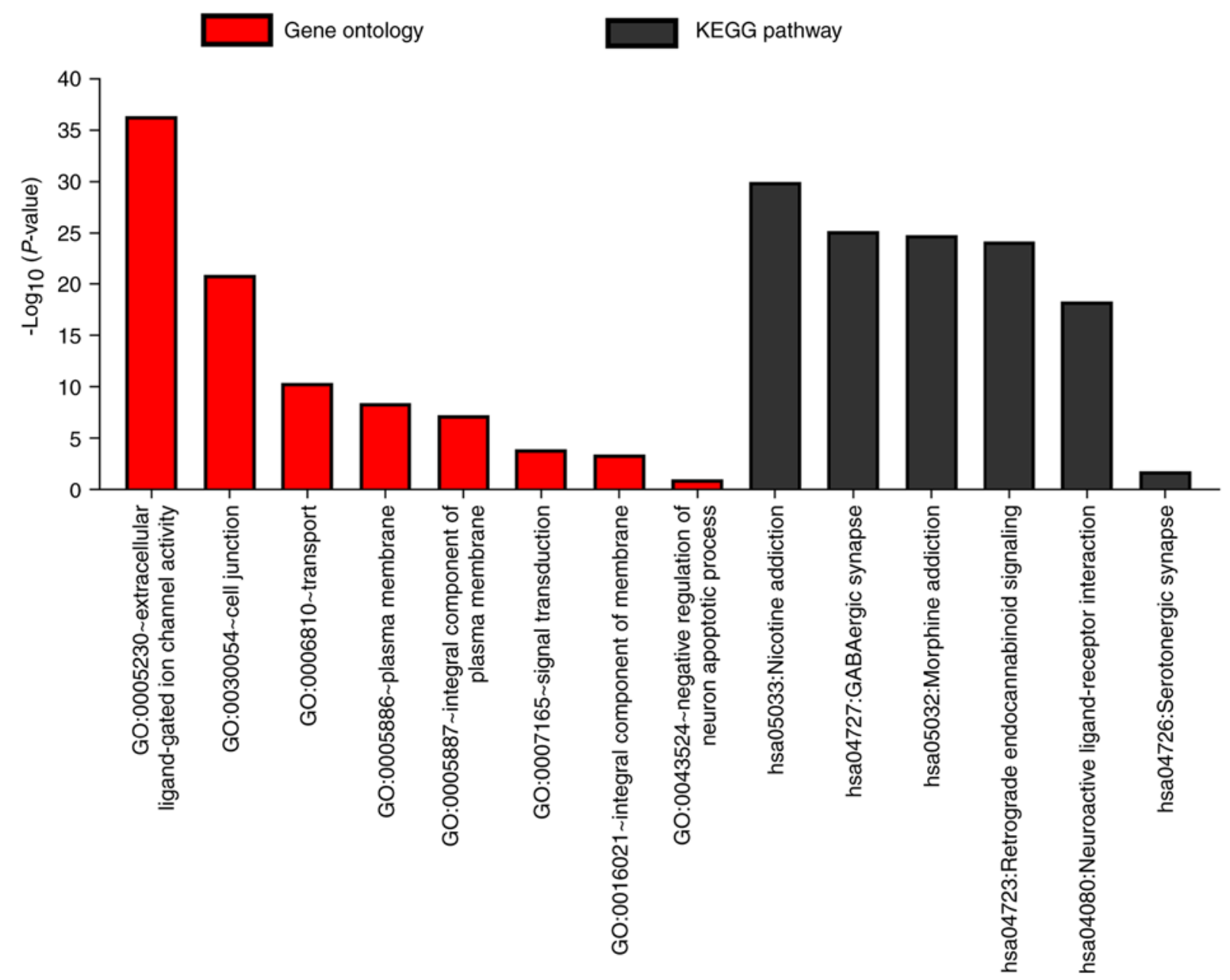

Figure 1. GO terms and KEGG analysis of all the $\gamma$-aminobutyric acid type A family genes using the Database for Explaining, Visualization and Integrated Discovery. GO, gene ontology; KEGG, Kyoto Encyclopedia of Genes and Genomes.

$\mathrm{HR}=1.807,95 \%$ CI 1.180-2.765; adjusted $\mathrm{P}=0.005, \mathrm{HR}=1.833$, 95\% CI 1.196-2.810 and adjusted $\mathrm{P}=0.034, \mathrm{HR}=1.578,95 \% \mathrm{CI}$ 1.036-2.405, respectively; Table III).
The nomogram of scoring risk included the expression levels of $G A B R B 1, G A B R D, G A B R P$ and $G A B R Q$ and predictive TNM stage, sex, age and 1-, 2-, 3, 4- and 5-year survival 
A

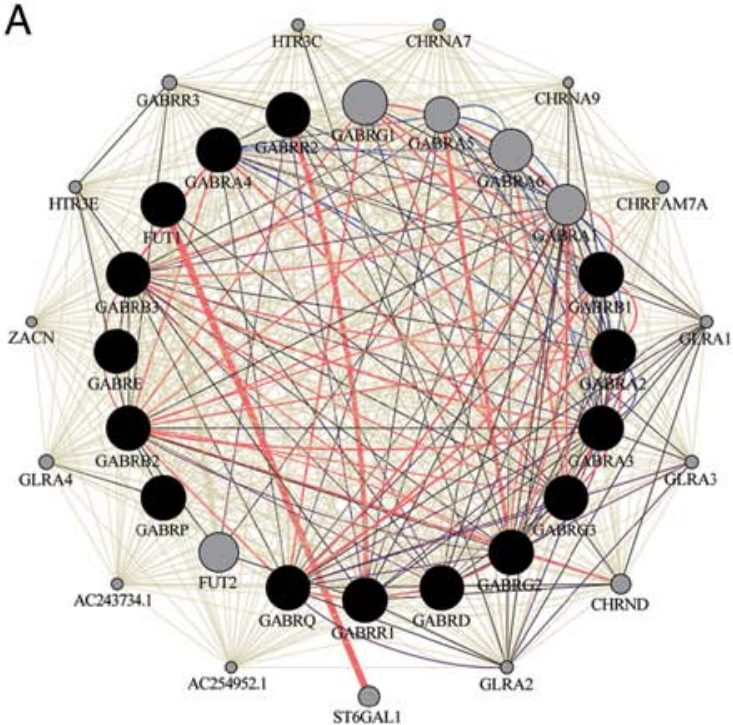

Shared protein domains —Predicted

Co-expression Co-localization

- Pathway
B

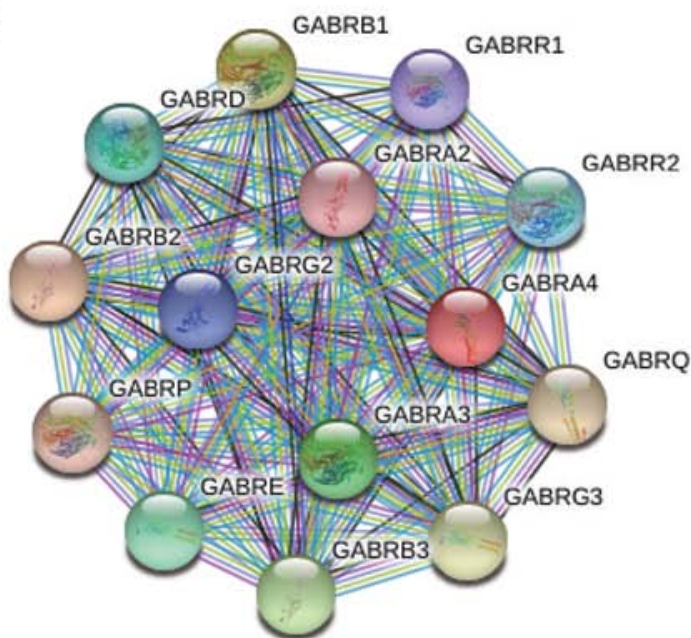

$\square$ Expermentally determined $=$ Texting
From curated databases $\simeq$ Gene neighborhood

Protein homology Co-expression

Figure 2. GeneMANIA and STRING analysis of $G A B A_{A}$ genes. (A) Gene interaction networks of $G A B A_{A}$ genes by GeneMANIA. (B) Protein-protein interation networks of $G A B A_{A}$ genes by STRING. GeneMANIA, Gene Multiple Association Network Integration Algorithm; STRING, search tool for the Retrieval of Interacting Genes/Proteins; $G A B A_{A}, \gamma$-Aminobutyric acid type A; $G A B R, \gamma$-aminobutyric acid type A receptor.

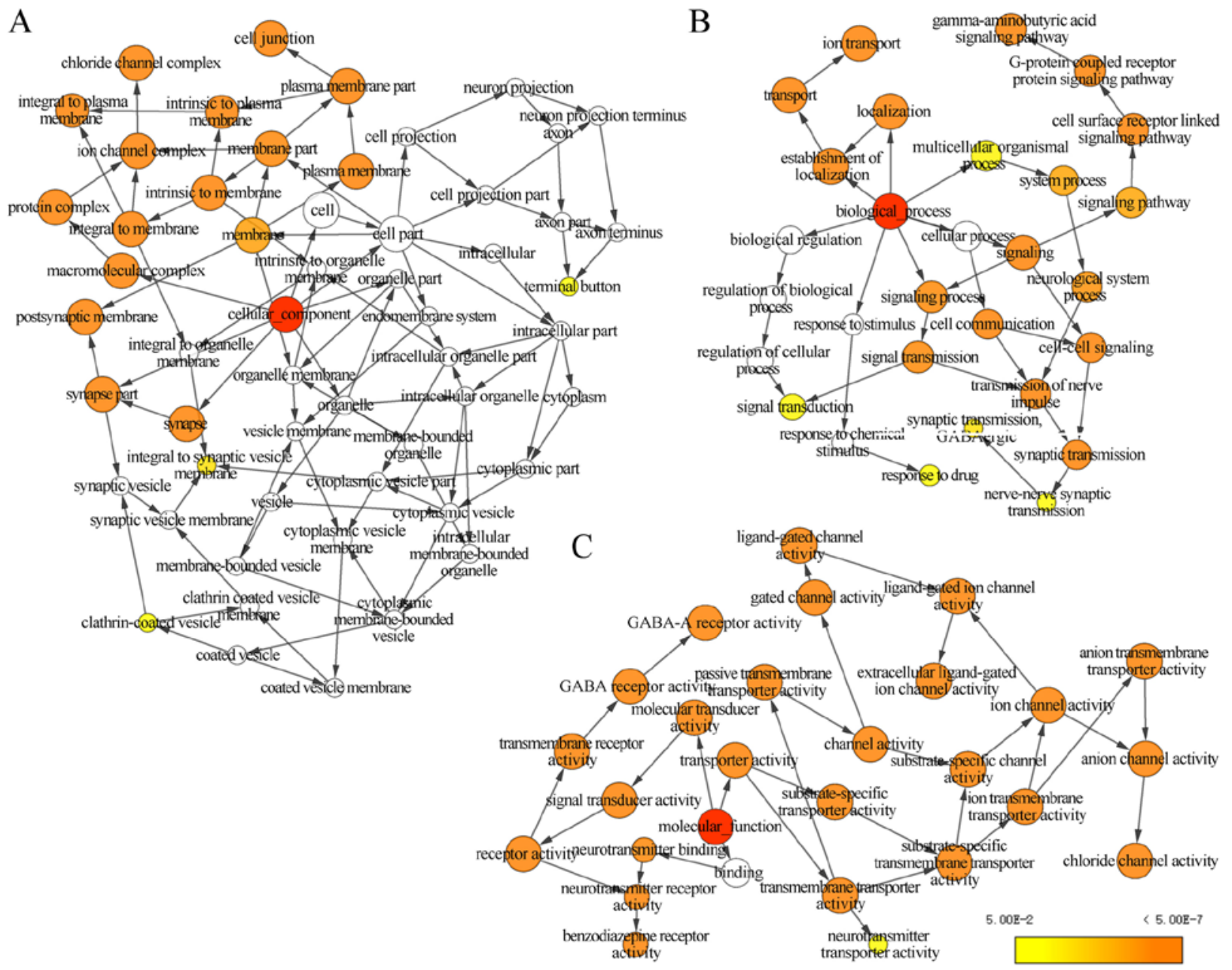

Figure 3. Biological Networks Gene Ontology analysis of $G A B A_{A}$ genes interaction networks. (A) Cellular component outcomes; (B) biological process outcomes; and (C) molecular function outcomes. $G A B R, \gamma$-aminobutyric acid type A receptor. 
Table II. Spearman's correlations test between GABRD expression and Tumor-Node-Metastasis stage in patients with colon adenocarcinoma in The Cancer Genome Atlas dataset.

\begin{tabular}{lcccc}
\hline Stage & Patients (n) & MST (days) & Spearman's Correlations coefficient & P-value \\
\hline I & 73 & NA & NA & NA \\
II & 167 & 2,821 & 0.090 & 0.164 \\
III & 126 & NA & 0.149 & $0.036^{\text {a }}$ \\
IV & 61 & 858 & 0.318 & $<0.001^{\text {b }}$ \\
Total & 427 & 2,821 & 0.174 & $<0.001^{\text {b }}$ \\
\hline
\end{tabular}

${ }^{\mathrm{a}} \mathrm{P}<0.05,{ }^{\mathrm{b}} \mathrm{P}<0.001$. MST, median survival time. GABR, $\gamma$-aminobutyric acid type A receptor.

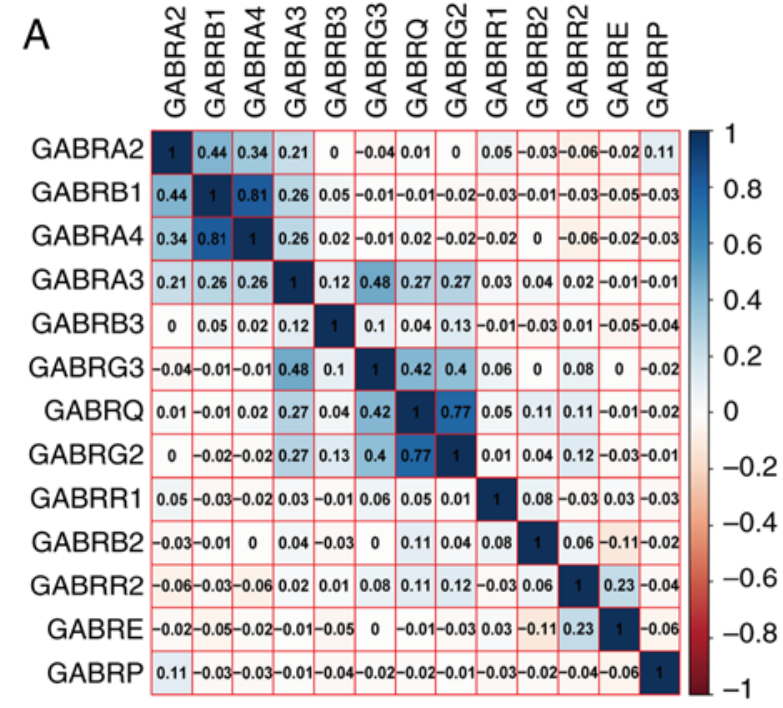

C

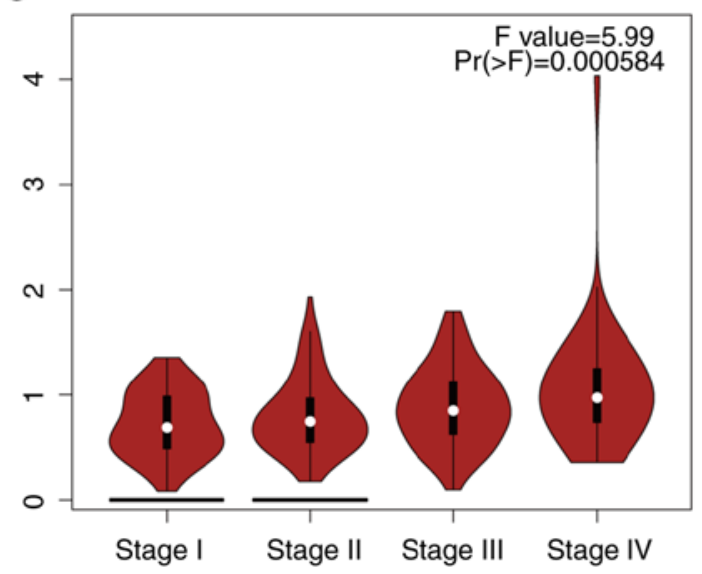

Figure 4. Pearson's correlation coefficients, scatterplots and violin plot of $G A B A_{A}$ genes. (A) Pearson's correlation coefficients for $\gamma$-aminobutyric acid type A gene expression levels. (B) Scatterplots for $G A B A_{A}$ gene family expression levels in The Cancer Genome Atlas. (C) Violin plot of $G A B R D$ expressions in Gene Multiple Association Network Integration Algorithm. ${ }^{* *} \mathrm{P}<0.001,{ }^{* * * *} \mathrm{P}<0.0001$. NS, not significant; GABR, $\gamma$-aminobutyric acid type A receptor.

rates (Fig. 8), it showed that the above risk factors contribute to the risk points, among which the age contribution is the smallest one and the stage contribution is the largest one, The higher the risk points, the lower the survival rates.

Effect of $G A B A_{A}$ genes expression combination on $O S$. Based on the survival analysis of $G A B A_{A}$ genes, GABRB1, GABRD,

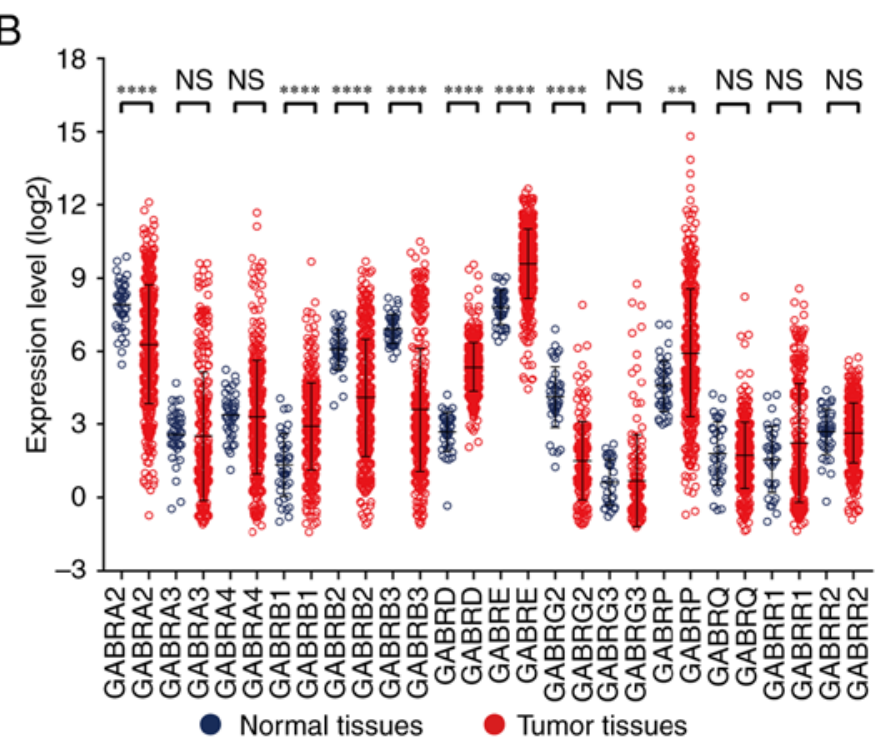

$G A B R P$ and $G A B R Q$ were selected as prognostic genes by multivariate survival analysis. The joint-effects of these four $G A B A_{A}$ genes on OS in patients with COAD were determined by the joint-effects model. According to the expression levels of $G A B R B 1, G A B R D, G A B R P$ and $G A B R Q$, different combinations for this analysis were generated (Tables IV-V). Log-rank tests were performed using Kaplan-Meier analysis to evaluate 
A
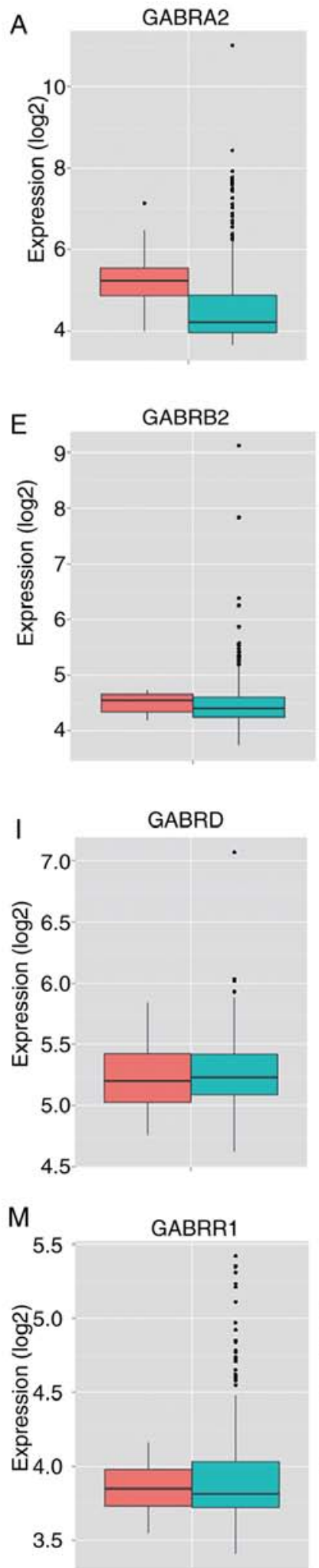
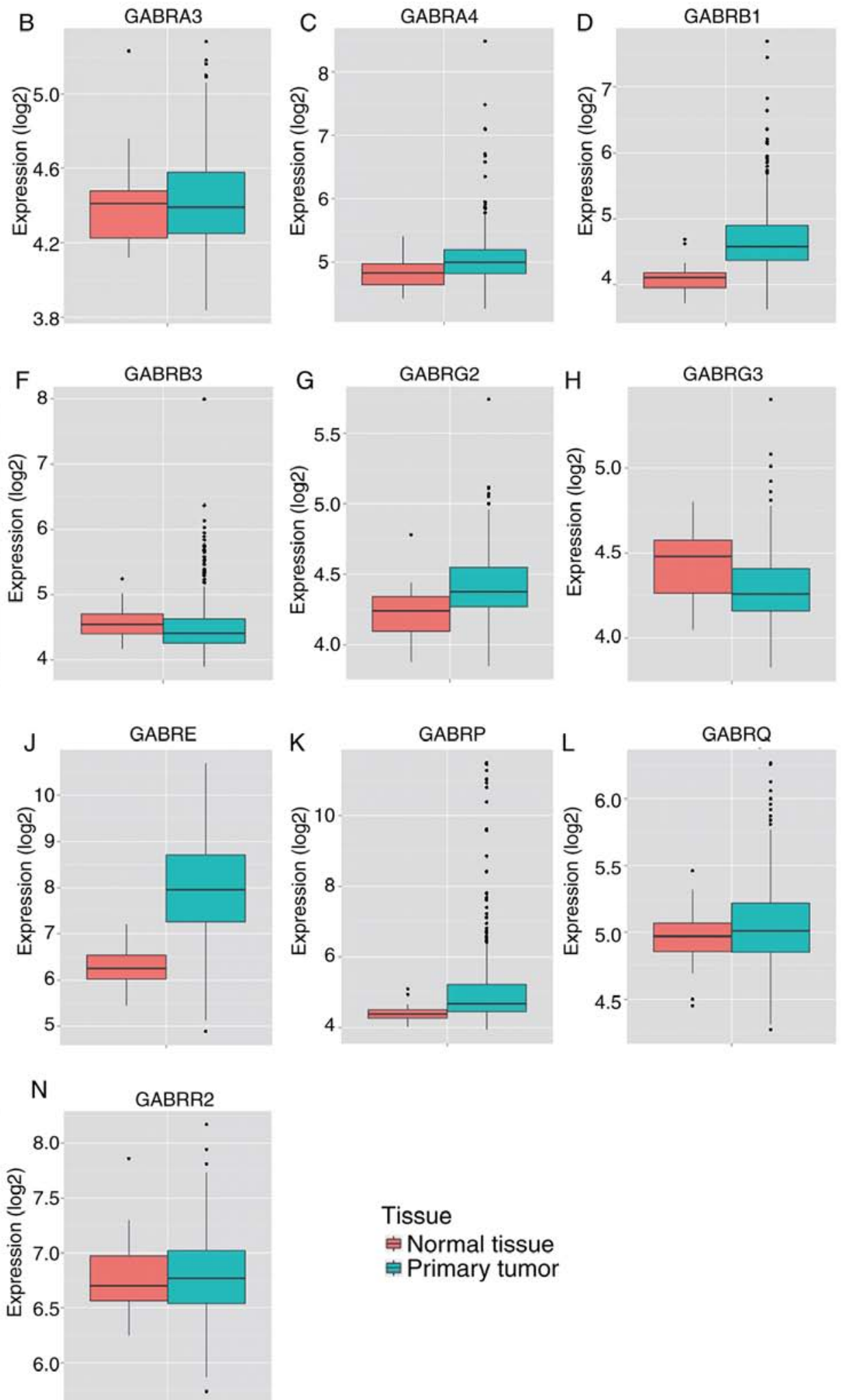

Tissue

自 Normal tissue

自 Primary tumor

Figure 5. Metabolic gene rapid visualizer boxplots of expression of $\gamma$-aminobutyric acid type A gene family in colon adenocarcinoma tumor tissues and adjacent normal tissues. Boxplots were shown for the expression levels of: (A) GABRA2; (B) GABRA3; (C) GABRA4; (D) GABRB1; (E) GABRB2; (F) GABRB3; (G) GABRG2; (H) GABRG3; (I) GABRD; (J) GABRE; (K) GABR; (L) GABRQ; (M) GABRRI; and (N) GABRR2. GABR, $\gamma$-aminobutyric acid type A receptor.

the effect of gene expression combinations on the prognosis of patients with COAD (Fig. 9). In the analysis of high expression levels of GABRB1, GABRD, GABRP and GABRQ, the combinations in groups $3,9,12,15,18, \mathrm{H}$ and $\mathrm{P}$ were highly correlated with poor OS (all $\mathrm{P}<0.05$; Table VI). Within the evaluation of low GABRB1, GABRD, GABRP and GABRQ expression levels, the combination of groups $1,7,10,13,16, \mathrm{~A}, \mathrm{E}, \mathrm{I}$ and $\mathrm{M}$ were highly correlated with favorable OS (all $\mathrm{P}<0.05$; Table VII). 

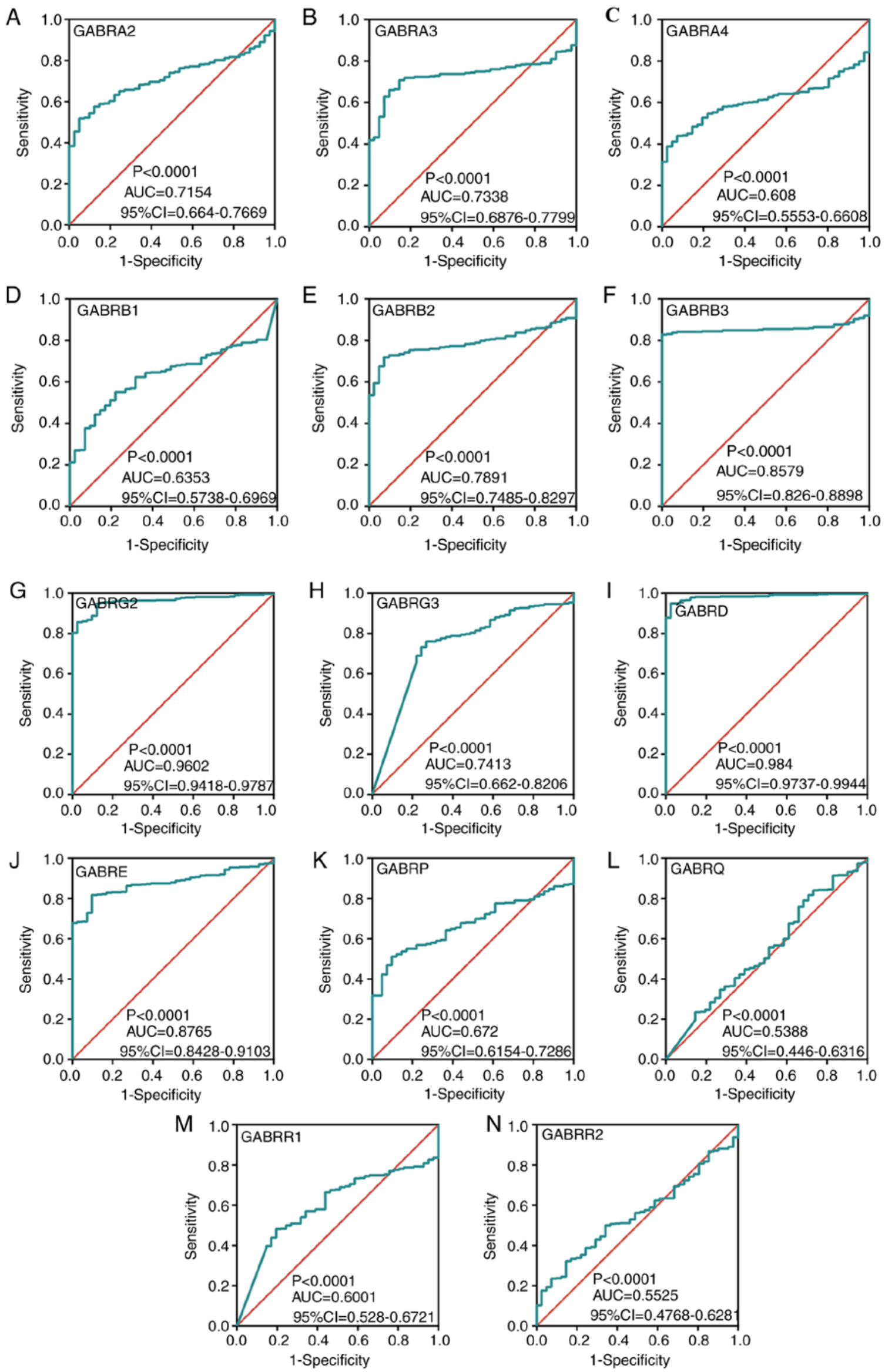

Figure 6. ROC curves of $\gamma$-aminobutyric acid type A genes for distinguishing colon adenocarcinoma tumor tissue and adjacent normal tissues in The Cancer Genome Atlas dataset. ROC curves of: (A) GABRA2; (B) GABRA3; (C) GABRA4; (D) GABRB1; (E) GABRB2; (F) GABRB3; (G) GABRG2; (H) GABRG3; (I) $G A B R D$; (J) GABRE; (K) GABRP; (L) GABRQ; (M) GABRRl; and (N) GABRR2. ROC, Receiver Operating Characteristic. GABR, $\gamma$-aminobutyric acid type A receptor; AUC, area under the curve; $\mathrm{CI}$, confidence interval. 
A

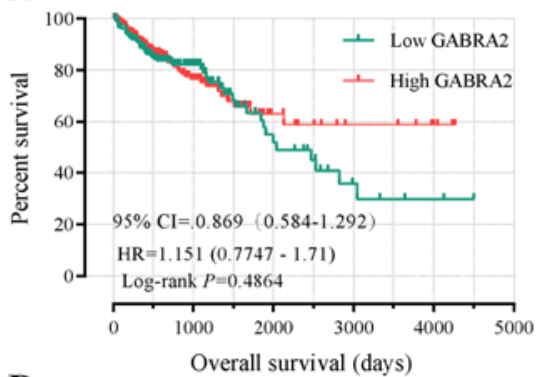

D

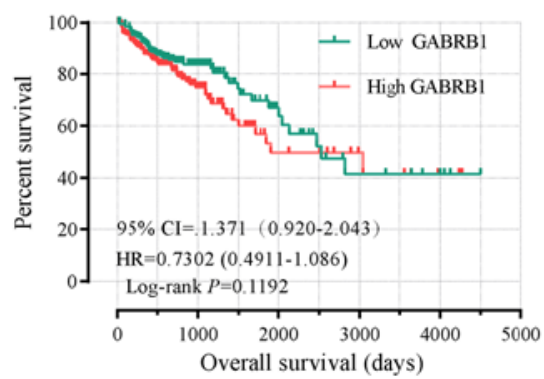

G

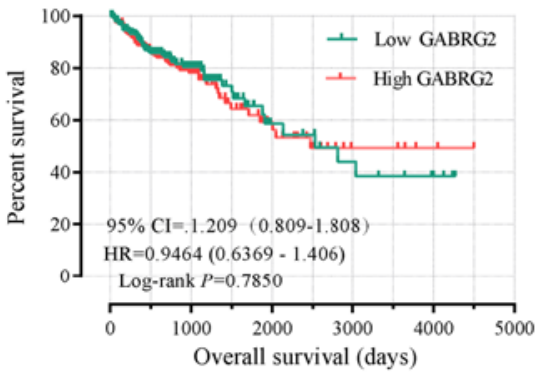

J

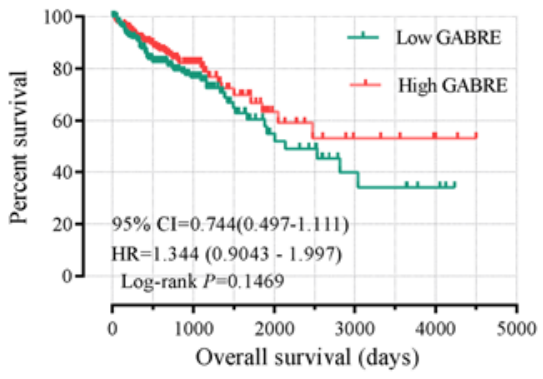

B

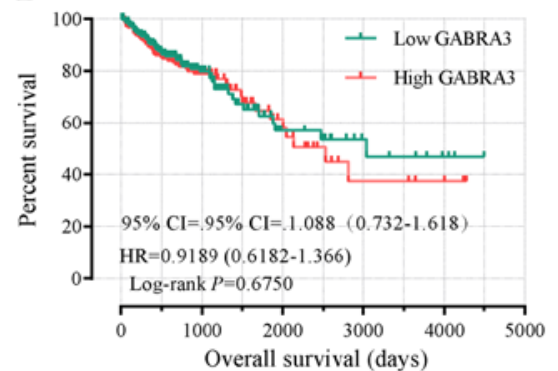

E

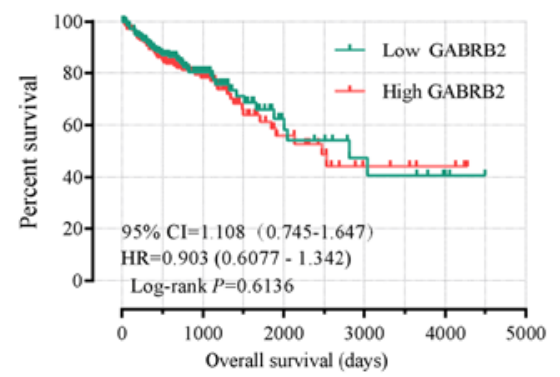

H

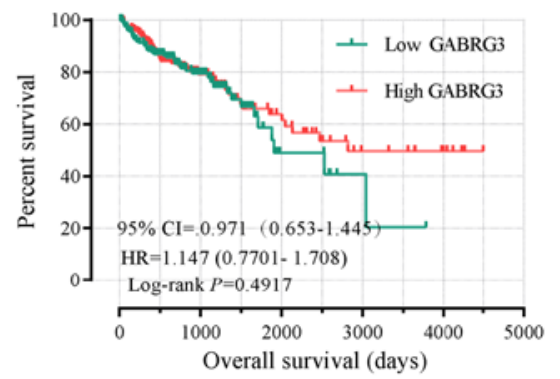

K

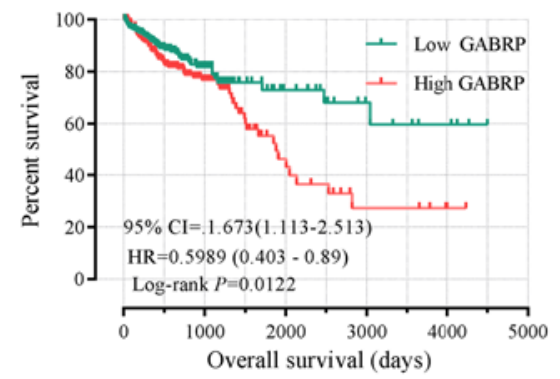

C

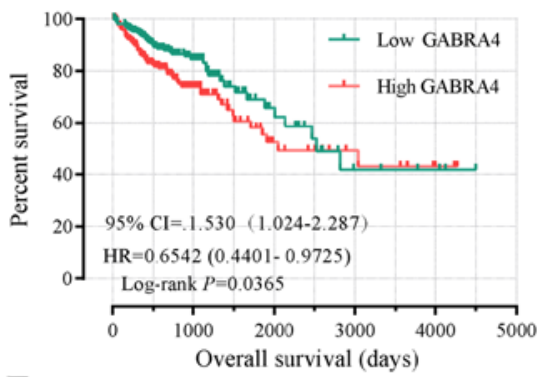

F

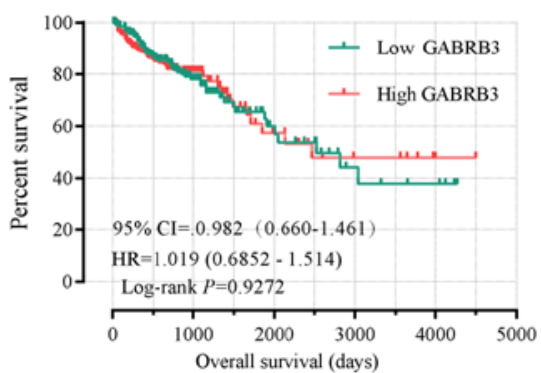

I

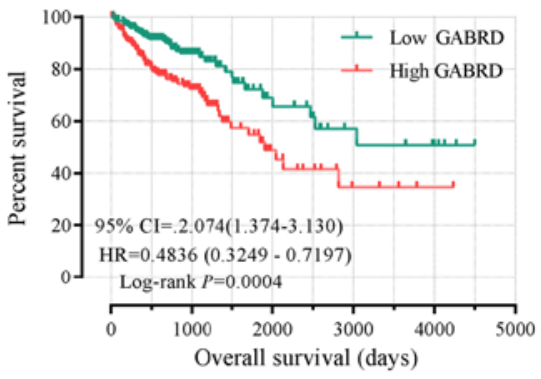

L

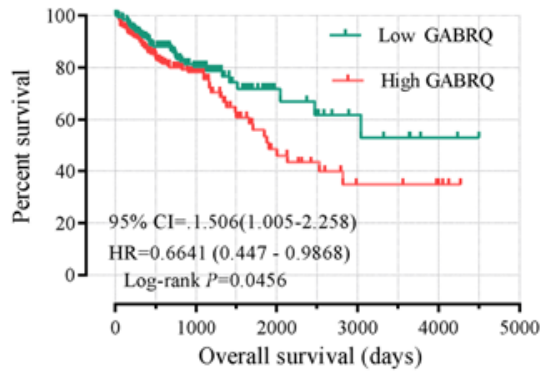

M

N
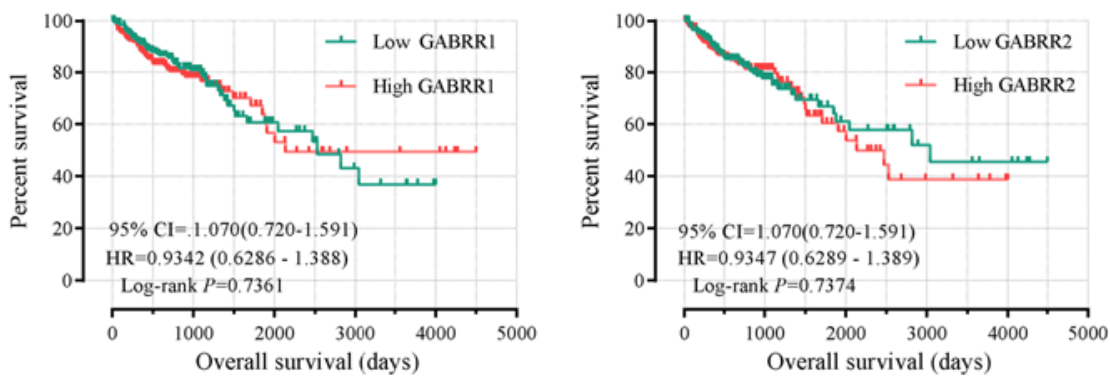

Figure 7. Kaplan-Meier survival curves for $\gamma$-aminobutyric acid type A genes in colon adenocarcinoma of The Cancer Genome Atlas cohort. Overall survival stratified by: (A) GABRA2; (B) GABRA3; (C) GABRA4; (D) GABRB1; (E) GABRB2; (F) GABRB3; (G) GABRG2; (H) GABRG3; (I) GABRD; (J) GABRE; (K) GABRP; (L) GABRQ; (M) GABRRI; (N) GABRR2. HR, hazard ratio; GABR, $\gamma$-aminobutyric acid type A receptor.

GSEA. GSEA of the prognostic genes GABRB1, GABRD, $G A B R P$ and $G A B R Q$ were performed in the TCGA cohorts
(Fig. 10). In the GSEA of KEGG pathways, the expression levels of the $G A B R D$ were associated with the chondroitin sulfate 
Table III. Prognostic survival analysis according to high or low expression of $\gamma$-aminobutyric acid type A receptor family genes in 438 patients with colon adenocarcinoma.

\begin{tabular}{|c|c|c|c|c|c|c|c|}
\hline Gene & Patients, $\mathrm{n}$ & Events $^{\mathrm{c}}$ & MST, days & $\begin{array}{l}\text { Crude HR } \\
(95 \% \mathrm{CI})\end{array}$ & $\begin{array}{l}\text { Crude } \\
\text { P-value }\end{array}$ & $\begin{array}{c}\text { Adjusted } \\
\text { HR }(95 \% \text { CI })\end{array}$ & $\begin{array}{l}\text { Adjusted } \\
\text { P-value }^{\mathrm{d}}\end{array}$ \\
\hline \multicolumn{8}{|c|}{ GABRA2 } \\
\hline Low & 219 & 52 & 2,047 & 1 & & 1 & \\
\hline High & 219 & 46 & NA & $0.869(0.584-1.292)$ & 0.487 & $0.792(0.525-1.196)$ & 0.267 \\
\hline \multicolumn{8}{|c|}{ GABRA3 } \\
\hline Low & 219 & 49 & 3,042 & 1 & & 1 & \\
\hline High & 219 & 49 & 2,532 & $1.088(0.732-1.618)$ & 0.675 & $1.099(0.730-1.654)$ & 0.651 \\
\hline \multicolumn{8}{|c|}{ GABRA4 } \\
\hline Low & 219 & 41 & 2,532 & 1 & & 1 & \\
\hline High & 219 & 57 & 2,047 & $1.530(1.024-2.287)$ & $0.038^{\mathrm{a}}$ & $1.499(0.989-2.271)$ & 0.056 \\
\hline \multicolumn{8}{|c|}{ GABRB1 } \\
\hline Low & 219 & 44 & 2,532 & 1 & & 1 & \\
\hline High & 219 & 54 & 1,910 & $1.371(0.920-2.043)$ & 0.121 & $1.517(1.001-2.297)$ & $0.049^{\mathrm{a}}$ \\
\hline \multicolumn{8}{|c|}{ GABRB2 } \\
\hline Low & 219 & 46 & 2,821 & 1 & & 1 & \\
\hline High & 219 & 52 & 2,475 & $1.108(0.745-1.647)$ & 0.614 & $1.343(0.887-2.033)$ & 0.163 \\
\hline \multicolumn{8}{|c|}{ GABRB3 } \\
\hline Low & 219 & 52 & 2,532 & 1 & & 1 & \\
\hline High & 219 & 46 & 2,475 & $0.982(0.660-1.461)$ & 0.927 & $1.170(0.776-1.765)$ & 0.454 \\
\hline \multicolumn{8}{|c|}{ GABRG2 } \\
\hline Low & 219 & 51 & 2,821 & 1 & & 1 & \\
\hline High & 219 & 47 & 2,475 & $1.209(0.809-1.808)$ & 0.355 & $1.296(0.854-1.967)$ & 0.223 \\
\hline \multicolumn{8}{|c|}{ GABRG3 } \\
\hline Low & 219 & 51 & 2,532 & 1 & & 1 & \\
\hline High & 219 & 47 & NA & $0.971(0.653-1.445)$ & 0.886 & $0.958(0.635-1.445)$ & 0.839 \\
\hline \multicolumn{8}{|c|}{ GABRD } \\
\hline Low & 219 & 36 & NA & 1 & & 1 & \\
\hline High & 219 & 62 & 1,910 & $2.074(1.374-3.130)$ & $0.001^{\mathrm{b}}$ & $1.807(1.180-2.765)$ & $0.006^{\mathrm{b}}$ \\
\hline \multicolumn{8}{|c|}{ GABRE } \\
\hline Low & 219 & 57 & 2,134 & 1 & & 1 & \\
\hline High & 219 & 41 & NA & $0.744(0.497-1.111)$ & 0.149 & $0.736(0.486-1.112)$ & 0.145 \\
\hline \multicolumn{8}{|c|}{ GABRP } \\
\hline Low & 219 & 38 & NA & 1 & & 1 & \\
\hline High & 219 & 60 & 1,881 & $1.673(1.113-2.513)$ & $0.013^{\mathrm{a}}$ & $1.833(1.196-2.810)$ & $0.005^{\mathrm{b}}$ \\
\hline \multicolumn{8}{|c|}{ GABRQ } \\
\hline Low & 219 & 39 & NA & 1 & & 1 & \\
\hline High & 219 & 59 & 1,910 & $1.506(1.005-2.258)$ & $0.047^{\mathrm{a}}$ & $1.578(1.036-2.405)$ & $0.034^{\mathrm{a}}$ \\
\hline \multicolumn{8}{|c|}{ GABRR1 } \\
\hline Low & 219 & 49 & 2,532 & 1 & & 1 & \\
\hline High & 219 & 49 & 2,134 & $1.070(0.720-1.591)$ & 0.736 & $1.079(0.717-1.625)$ & 0.714 \\
\hline \multicolumn{8}{|c|}{ GABRR2 } \\
\hline Low & 219 & 49 & 3,042 & 1 & & 1 & \\
\hline High & 219 & 49 & 2,134 & $1.070(0.720-1.591)$ & 0.738 & $1.259(0.833-1.902)$ & 0.274 \\
\hline
\end{tabular}

${ }^{\mathrm{a} P}<0.05 .{ }^{\mathrm{b}} \mathrm{P}<0.01 .{ }^{\mathrm{c}}$ Number of final events. ${ }^{\mathrm{d}}$ Adjusted for tumor stage. HR, hazard ratio; CI, confidence interval; MST, median survival time; GABAA, $\gamma$-aminobutyric acid type A.

pathway (Fig. 10H) and GABRP was associated with the intes- hematopoietic cell lineage, the natural killer cell mediated tinal immune network for Immunoglobulin A (IGA) production, cytotoxicity pathway, sphingolipid metabolism (Fig. 10I-L). GO 
Points

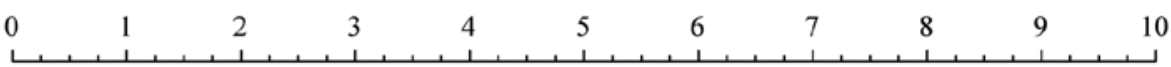

Sex

$$
\begin{aligned}
& \text { Male } \\
& \text { Female }
\end{aligned}
$$

age

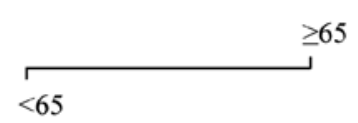

Stage

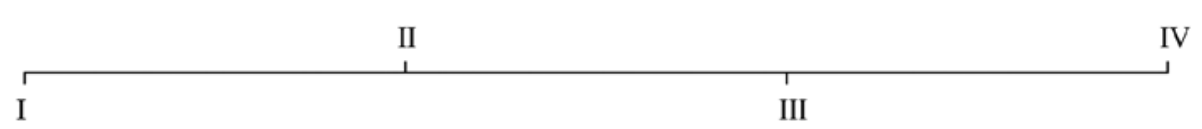

\section{GABRB1}

GABRD

GABRP

GABRQ

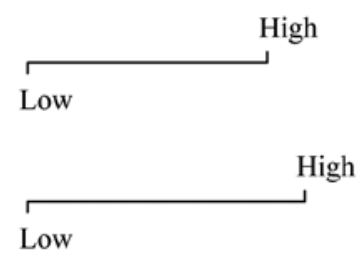

Low

High

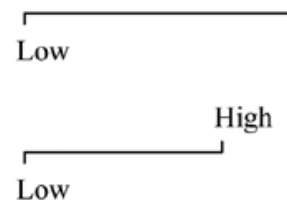

Total Points

\begin{tabular}{lllllllllllll}
\hline & 2 & 4 & 6 & 8 & 10 & 12 & 14 & 16 & 18 & 20 & 22
\end{tabular}

1-year overall survival

\begin{tabular}{llllllll}
\hline & & & & & & 1 & \\
0.95 & & 0.9 & 0.85 & 0.8 & 0.750 .7 & 0.6 & 0.5
\end{tabular}

2-year overall survival

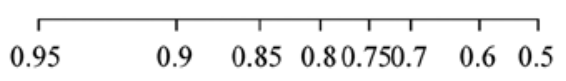

3-year overall survival

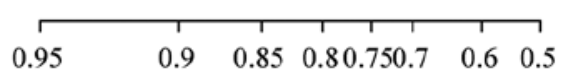

4-year overall survival

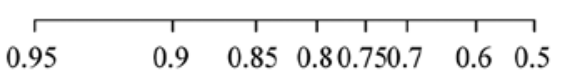

5-year overall survival

\begin{tabular}{llllllll}
\hline & & & & 1 & 1 & 1 & 1 \\
0.95 & & 0.9 & 0.85 & 0.80 .750 .7 & 0.6 & 0.5
\end{tabular}

Figure 8. Nomogram of OS-associated GABRB1, GABRD, GABRP, GABRQ and clinical factors. $G A B R$, $\gamma$-aminobutyric acid type A receptor.

function enriched examination demonstrated that that $G A B R D$ expression levels were associated with the cell matrix adhesion, integrin, angiogenesis, endothelial growth factor, endothelial migration regulation, and so on (Fig. 10A-G); whereas GABRBI and $G A B R Q$ had no significant outcomes.

\section{Discussion}

In the present study, the diagnostic and prognosis value of the $G A B A_{A}$ family genes based on TCGA database were investigated. The results of ROC curves showed that expression levels of GABRB3, GABRG2, GABRD and GABRE had high values to predict the occurrence of colon cancer, among them, GABRD was associated with COAD stage and may have value as an early diagnostic index of COAD. The results were roughly the same as verified in MERAV and Vertical scatterplots. Low expression levels of GABRB1, GABRD, GABRP and $G A B R Q$ were associated with favorable COAD OS and the nomogram indicated these four genes had different degrees of influence on the prognosis of the patients, high expression 
Table IV. Grouping according to combination of 2 genes in $G A B R B 1, G A B R D, G A B R P$ and $G A B R Q$.

\begin{tabular}{cc}
\hline Group & Combination \\
\hline 1 & Low GABRB1 + Low GABRD \\
2 & Low GABRB1 + High GABRD \\
& High GABRB1 + Low GABRD \\
& High GABRD + Low GABRP \\
3 & High GABRB1 + High GABRD \\
4 & Low GABRB1 + Low GABRP \\
5 & Low GABRB1 + High GABRP \\
& High GABRB1 + Low GABRP \\
& High GABRD + Low GABRQ \\
6 & High GABRB1 + High GABRP \\
7 & Low GABRB1 + Low GABRQ \\
8 & Low GABRB 1 + High GABRQ \\
& High GABRB1 + Low GABRQ \\
9 & High GABRP + Low GABRQ \\
10 & High GABRB1 + High GABRQ \\
11 & Low GABRD + Low GABRP \\
12 & Low GABRD + High GABRP \\
13 & High GABRD + High GABRP \\
14 & Low GABRD + Low GABRQ \\
15 & Low GABRD + High GABRQ \\
16 & High GABRD + High GABRQ \\
17 & Low GABRP + Low GABRQ \\
18 & Low GABRP + High GABRQ \\
& High GABRP + High GABRQ
\end{tabular}

GABR, $\gamma$-aminobutyric acid type A receptor.

of GABRB1, GABRD, GABRP have high contribution to the risk score than high expression of GABRQ. In the functional evaluation of GO and KEGG, it was found that the functions of the $G A B A_{A}$ gene family were significantly enriched in cell junction, integral component of membrane, signal transduction, integral component of plasma membrane.

$G A B A_{A}$ receptors have the same structure with nicotinic acetylcholine receptors, the 5-hydroxytryptamine type 3 receptor and zinc-activated channel, all with pentameric structures and belonging to the agonist-gated ion channel superfamily (29). STRING results showed that obvious gene fusions, gene co-occurrence and co-expression between $G A B A_{A}$ genes. Pearson correlation coefficient analysis showed that there was a correlation between the expression levels of some genes in the $G A B A_{A}$ family, especially between $G A B R B I$ and $G A B A 4$, and $G A B R Q$ and $G A B R G 2$.

The $G A B A_{A}$ family genes also serve a role in several types of cancer, Gumireddy et al (30) found that the high expression levels of GABRA3 were inversely proportional to the survival rate of patients with breast cancer and that GABRA3 activated the AKT pathway which promoted the migration, invasion and metastasis of breast cancer cells. Therefore, GABRA4 might serve a role in COAD, which requires further study. Bautista et al (31) observed that the expression levels of GABRA6 in tumor initiating stem cells (TISCS) and hepatocellular carcinoma (HCC) were reduced, whereas the expression levels of $G A B R G 3$ were abundant in TISCS and limited in HCC. A previous study showed that the specific activation of $G A B A_{A}$ receptor decreased cell activity, induced apoptosis and inhibited the growth and survival signal pathway of neuroblastoma cells (32). Chen et al (33) found that $G A B A_{A}$ receptor could inhibit the migration and invasion of human hepatocellular carcinoma cells and Minuk et al (34) reported downregulated expression of the GABRB3 receptor in liver tissue of human hepatocellular carcinoma, which was consistent with COAD in the present study. Takehara et al (35) found that $G A B A$ promoted the growth of pancreatic cancer by expressing $G A B A_{A}$ receptor $G A B R P$ subunit. Zhang et al showed that RNA binding protein nova 1 and GABRG2 interacted in the central nervous system and in liver cancer. Nova 1 , as a potential mechanism of oncogene, might interact with $G A B R G 2$ (36). To sum up, the $G A B A_{A}$ family plays an important role in many cancer types, Nevertheless, the correlation between $G A B A_{A}$ family and COAD is unclear. Here, we use the TCGA database to study the correlation of GABAA gene family expression with diagnosis and prognosis.

GSEA analysis showed that $G A B R D$ was associated with cell matrix adhesion and integrin binding. Cell adhesion is an important cellular process that could lead to cancer $(37,38)$. As the main receptor of cell matrix adhesion, integrin exists on the surface of tumor and stroma cells, which had a profound impact on cancer cell's ability to survive in a specific location, cell adhesion and integrin can worked together to lead to apoptosis (39). In addition, integrin also serves a role in promoting the phenotype of tumor cells (40). The present study also suggested that $G A B R D$ was significantly associated with angiogenesis and endothelial migration regulation in GSEA. These factors serve a role in tumor invasion and migration (41-43). In addition, tumor angiogenesis is also one of the markers of tumor progression and the increase of tumor microvessel density is an index of poor prognosis (44). Park et al reported that human $\gamma$-aminobutyrate type A receptor-binding protein (GABARBP) could inhibit angiogenesis by directly binding to vascular endothelial growth factor receptor 2 (VEGFR-2) to inhibit the phosphorylation of PI3K/AKT pathway related proteins (45). GABARBP served a role in regulating the activity of $G A B A_{A}$ receptor, a key participant in intracellular trafficking in all the $G A B A_{A}$ receptors (46-48). Therefore, the $G A B A A$ family genes may affect angiogenesis through regulating GABRBP, which needs to be verified in future experiments. In the present study, KEGG pathway analysis showed that $G A B R D$ was associated with chondroitin sulfate synthesis. Chondroitin sulfate serves a role in cancer metastasis and chondroitin sulfate-E negatively adjusted breast cancer cell motility through the Wnt/ $\beta$-catenin-Collagen I axis (49,50).

In the present study, it was observed that the expression of GABRD mRNA in adjacent tissues was significantly lower compared with COAD tumor tissues, which was consistent with the results of a previous study (14). KEGG pathway analysis of the present study showed that GABRP was associated with intestinal immune network for IGA production, hematopoietic cell lineage, natural killer (NK) cell mediated cytotoxicity and sphingolipid metabolism. In previous studies, people with IgA deficiency were found to have a moderately increased risk of 
Table V. Grouping according to combination of 3 genes in GABRB1, GABRD, GABRP and GABRQ.

\begin{tabular}{llll}
\hline Group & \multicolumn{1}{c}{ Combination } & Group & Combination \\
\hline A & Low GABRB1 + Low GABRD + Low GABRP & I & Low GABRB1 + Low GABRP + Low GABRQ \\
B & Low GABRB1 + High GABRD + Low GABRP & J & Low GABRB1 + High GABRP + Low GABRQ \\
& Low GABRB1 + Low GABRD + High GABRP & & Low GABRB1 + Low GABRP + High GABRQ \\
& High GABRB1 + Low GABRD + Low GABRP & & High GABRB1 + Low GABRP + Low GABRQ \\
C & High GABRB1 + High GABRD + Low GABRP & K & High GABRB1 + High GABRP + Low GABRQ \\
& High GABRB1 + Low GABRD + High GABRP & & High GABRB1 + Low GABRP + High GABRQ \\
& Low GABRB1 + High GABRD + High GABRP & & Low GABRB1 + High GABRP + High GABRQ \\
D & High GABRB1 + High GABRD + High GABRP & L & High GABRB1 + High GABRP + High GABRQ \\
E & Low GABRB1 + Low GABRD + Low GABRQ & M & Low GABRD + Low GABRP + Low GABRQ \\
F & Low GABRB1 + High GABRD + Low GABRQ & N & Low GABRD + High GABRP + Low GABRQ \\
& Low GABRB1 + Low GABRD + High GABRQ & & Low GABRD + Low GABRP + High GABRQ \\
& High GABRB1 + Low GABRD + Low GABRQ & & High GABRD + Low GABRP + Low GABRQ \\
G & High GABRB1 + High GABRD + Low GABRQ & O & High GABRD + High GABRP + Low GABRQ \\
& High GABRB1 + Low GABRD + High GABRQ & & High GABRD + Low GABRP + High GABRQ \\
& Low GABRB1 + High GABRD + High GABRQ & & Low GABRD + High GABRP + High GABRQ \\
H & High GABRB1 + High GABRD + High GABRQ & P & High GABRD + High GABRP + High GABRQ
\end{tabular}

GABR, $\gamma$-aminobutyric acid type A receptor; 1-18, 2 selected genes groups; A-P, 3 selected genes groups.
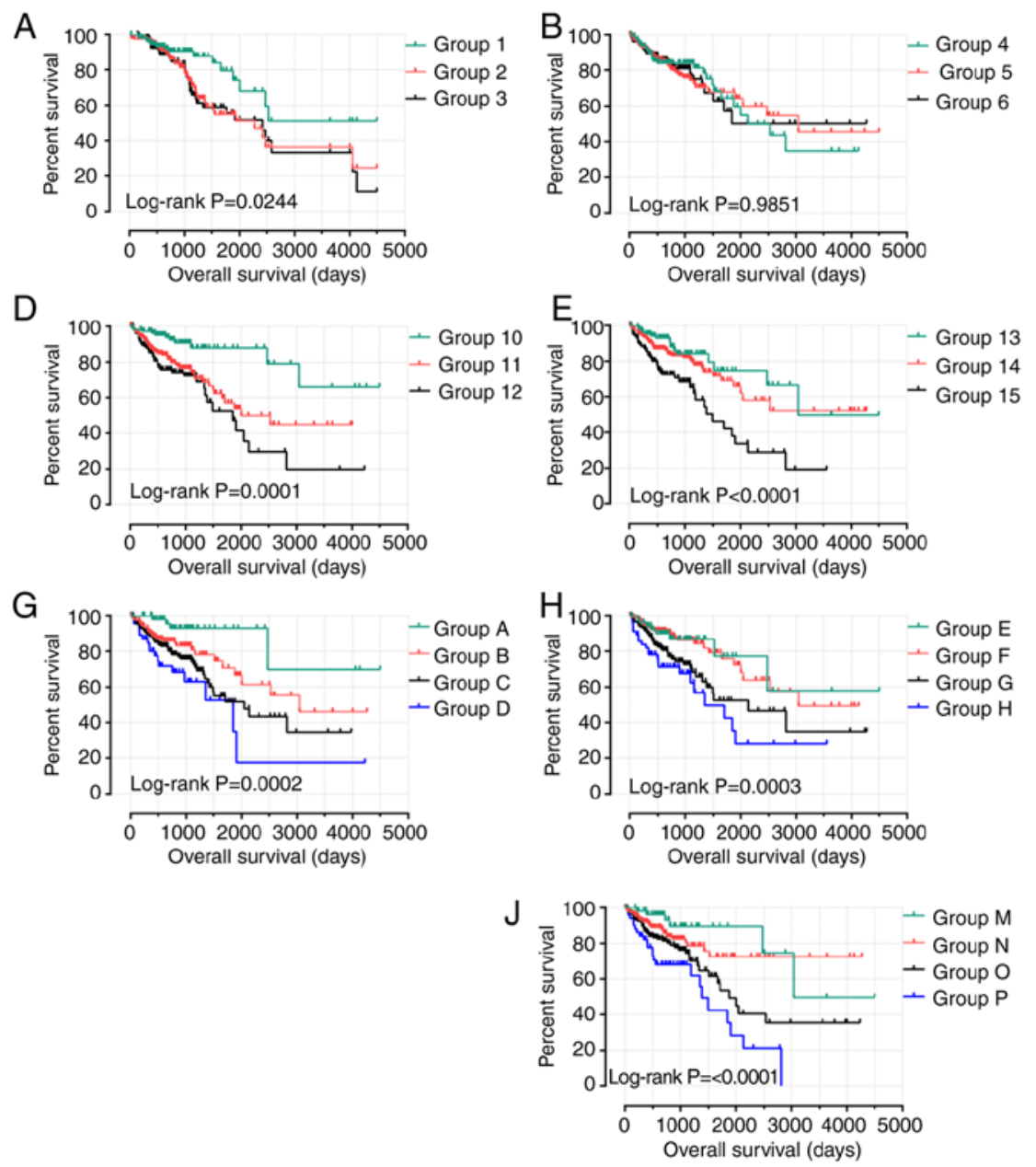
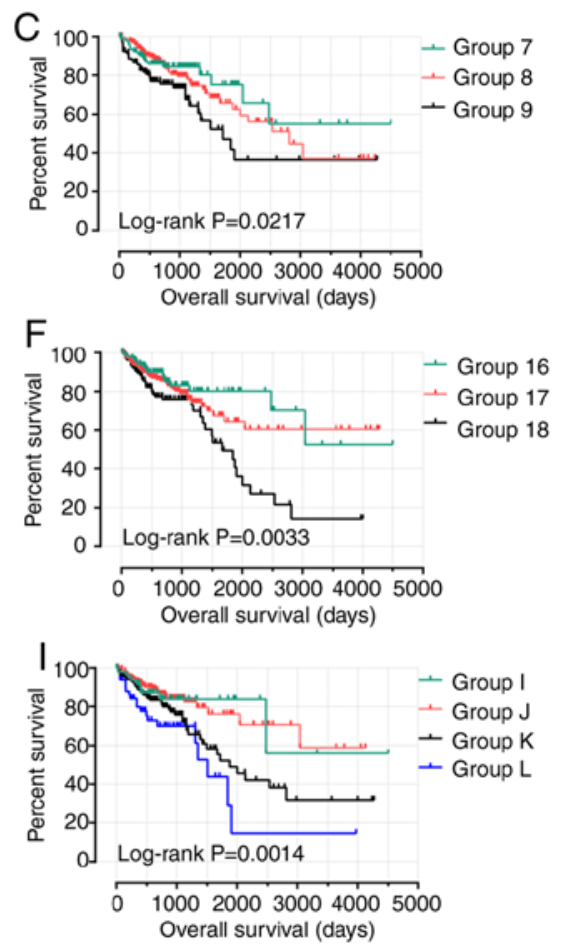

Overall survival (days)

Figure 9. Survival curves for joint-effects analysis of the combination of $G A B A A_{A}$ genes in patients with colon adenocarcinoma in TCGA dataset. Joint-effects analysis of (A) GABRBI and GABRD; (B) GABRBI and GABRP; (C) GABRBI and GABRQ; (D) GABRD and GABRP; (E) GABRD and GABRQ; (F) GABRP and $G A B R Q$; (G) GABRB1, GABRD and GABRP; (H) GABRB1, GABRD and GABRQ; (I) GABRB1, GABRP and GABRQ; (J) GABRD, GABRP and GABRQ. $G A B R, \gamma$-aminobutyric acid type A receptor; TCGA, the Cancer Genome Atlas. 
Table VI. Joint analysis of the prognostic value of 2-gene combinations in GABRB1, GABRD, GABRP and GABRQ expression of patients with colon adenocarcinoma.

\begin{tabular}{|c|c|c|c|c|c|c|}
\hline Group & Patients & MST, days & Crude P-value & Crude HR & Adjusted P-value & Adjusted HR $(95 \% \text { CI })^{\mathrm{d}}$ \\
\hline 1 & 115 & 1 & $0.003^{\mathrm{b}}$ & 1 & $0.007^{\mathrm{b}}$ & 1 \\
\hline 2 & 208 & 2,821 & $0.021^{\mathrm{a}}$ & $1.947(1.105-3.431)$ & $0.020^{\mathrm{a}}$ & $2.009(1.118-3.611)$ \\
\hline 3 & 115 & 1,849 & $0.001^{\mathrm{b}}$ & $2.814(1.551-5.104)$ & $0.002^{\mathrm{b}}$ & $2.712(1.460-5.039)$ \\
\hline 4 & 112 & 2,134 & 0.985 & 1 & 0.921 & 1 \\
\hline 5 & 214 & 3,042 & 0.865 & $1.042(0.648-1.676)$ & 0.966 & $1.011(0.616-1.659)$ \\
\hline 6 & 112 & 1 & 0.947 & $1.019(0.587-1.768)$ & 0.720 & $1.110(0.628-1.962)$ \\
\hline 7 & 110 & 1 & $0.024^{\mathrm{a}}$ & 1 & $0.011^{\mathrm{a}}$ & 1 \\
\hline 8 & 218 & 2,821 & 0.506 & $1.200(0.702-2.051)$ & 0.263 & $1.381(0.784-2.431)$ \\
\hline 9 & 110 & 1,711 & $0.016^{\mathrm{a}}$ & $1.994(1.137-3.497)$ & $0.005^{\mathrm{b}}$ & $2.333(1.287-4.231)$ \\
\hline 10 & 112 & 1 & $0.000^{\mathrm{c}}$ & 1 & $0.001^{\mathrm{b}}$ & 1 \\
\hline 11 & 214 & 2,532 & $0.001^{\mathrm{b}}$ & $2.936(1.530-5.634)$ & $0.006^{\mathrm{b}}$ & $2.620(1.318-5.207)$ \\
\hline 12 & 112 & 1,849 & $0.000^{\mathrm{c}}$ & $4.026(2.042-7.937)$ & $0.000^{\mathrm{c}}$ & $4.033(1.967-8.270)$ \\
\hline 13 & 110 & 3,042 & 0.000 & 1 & $0.001^{\mathrm{b}}$ & 1 \\
\hline 14 & 218 & 1 & 0.249 & $1.402(0.790-2.490)$ & 0.332 & $1.342(0.741-2.431)$ \\
\hline 15 & 110 & 1,493 & $0.000^{\mathrm{c}}$ & $2.934(1.639-5.255)$ & $0.002^{\mathrm{b}}$ & $2.658(1.453-4.863)$ \\
\hline 16 & 110 & 1 & $0.001^{\mathrm{b}}$ & 1 & $0.000^{\mathrm{c}}$ & 1 \\
\hline 17 & 218 & 1 & 0.332 & $1.342(0.741-2.431)$ & 0.249 & $1.402(0.790-2.490)$ \\
\hline 18 & 110 & 1,661 & $0.002^{\mathrm{b}}$ & $2.658(1.453-4.863)$ & $0.000^{\mathrm{c}}$ & $2.934(1.639-5.255)$ \\
\hline
\end{tabular}

${ }^{\mathrm{a}} \mathrm{P}<0.05,{ }^{\mathrm{b}} \mathrm{P}<0.01,{ }^{\mathrm{c}} \mathrm{P}<0.001 .1-18,2$ selected genes groups. ${ }^{\mathrm{d}}$ Adjustment for TNM stage. GABR, $\gamma$-aminobutyric acid type A receptor; MST, median survival time; HR, hazard ratio; CI, confidence interval; GABR, $\gamma$-aminobutyric acid type A receptor.

Table VII. Joint analysis of the prognostic value of 3 genes combination in $G A B R B 1, G A B R D, G A B R P$ and $G A B R Q$ expression of patients with colon adenocarcinoma.

\begin{tabular}{|c|c|c|c|c|c|c|}
\hline Group & Patients & MST, days & Crude P-value & Crude HR & Adjusted P-value & Adjusted HR $(95 \% \mathrm{CI})^{\mathrm{d}}$ \\
\hline A & 61 & 1 & $0.001^{\mathrm{b}}$ & 1 & $0.000^{\mathrm{c}}$ & 1 \\
\hline $\mathrm{B}$ & 148 & 3,042 & 0.000 & $0.130(0.044-0.386)$ & $0.000^{\mathrm{c}}$ & $0.103(0.030-0.357)$ \\
\hline $\mathrm{C}$ & 178 & 2,047 & $0.007^{\mathrm{b}}$ & $0.439(0.241-0.801)$ & $0.002^{\mathrm{b}}$ & $0.374(0.201-0.695)$ \\
\hline $\mathrm{D}$ & 51 & 1,849 & 0.127 & $0.649(0.373-1.131)$ & 0.060 & $0.581(0.330-1.023)$ \\
\hline $\mathrm{E}$ & 57 & 1 & $0.001^{\mathrm{b}}$ & 1 & $0.000^{\mathrm{c}}$ & 1 \\
\hline $\mathrm{F}$ & 164 & 3,042 & 0.864 & $1.072(0.487-2.359)$ & 0.945 & $0.971(0.418-2.255)$ \\
\hline G & 158 & 2,134 & $0.047^{\mathrm{a}}$ & $2.159(1.011-4.607)$ & $0.030^{\mathrm{a}}$ & $2.439(1.089-5.462)$ \\
\hline $\mathrm{H}$ & 59 & 1,348 & $0.007^{\mathrm{b}}$ & $3.067(1.365-6.892)$ & $0.028^{\mathrm{a}}$ & $2.626(1.110-6.231)$ \\
\hline I & 52 & 1 & $0.002^{\mathrm{b}}$ & 1 & $0.000^{\mathrm{c}}$ & 1 \\
\hline $\mathrm{J}$ & 168 & 1 & $0.015^{\mathrm{a}}$ & $0.360(0.157-0.823)$ & $0.003^{\mathrm{b}}$ & $0.249(0.099-0.629)$ \\
\hline $\mathrm{K}$ & 165 & 1,881 & $0.001^{\mathrm{b}}$ & $0.349(0.193-0.632)$ & $0.000^{\mathrm{c}}$ & $0.304(0.166-0.556)$ \\
\hline $\mathrm{L}$ & 53 & 1,503 & 0.119 & $0.651(0.380-1.116)$ & 0.046 & $0.573(0.332-0.989)$ \\
\hline M & 59 & 3,042 & $0.000^{\mathrm{c}}$ & 1 & $0.000^{\mathrm{c}}$ & 1 \\
\hline $\mathrm{N}$ & 155 & 1 & 0.250 & $1.685(0.693-4.096)$ & 0.106 & $2.222(0.845-5.843)$ \\
\hline $\mathrm{O}$ & 170 & 1,881 & $0.014^{\mathrm{a}}$ & $2.914(1.240-6.852)$ & $0.026^{\mathrm{a}}$ & $2.883(1.136-7.318)$ \\
\hline $\mathrm{P}$ & 54 & 1,381 & $0.000^{\mathrm{c}}$ & $5.003(2.034-12.307)$ & $0.000^{\mathrm{c}}$ & $7.157(2.689-19.053)$ \\
\hline
\end{tabular}

${ }^{\mathrm{a}} \mathrm{P}<0.05,{ }^{\mathrm{b}} \mathrm{P}<0.01,{ }^{\mathrm{c}} \mathrm{P}<0.001 .{ }^{\mathrm{d}}$ Adjustment for TNM stage. GABR, $\gamma$-aminobutyric acid type A receptor; MST, median survival time; HR, hazard ratio; $\mathrm{CI}$, confidence interval.

cancer, especially gastrointestinal cancer (51). NK cells also play an important role in mediating immune surveillance for human cancer (52). As the structural molecules of cell membranes, sphingolipids play an important role in maintaining barrier 

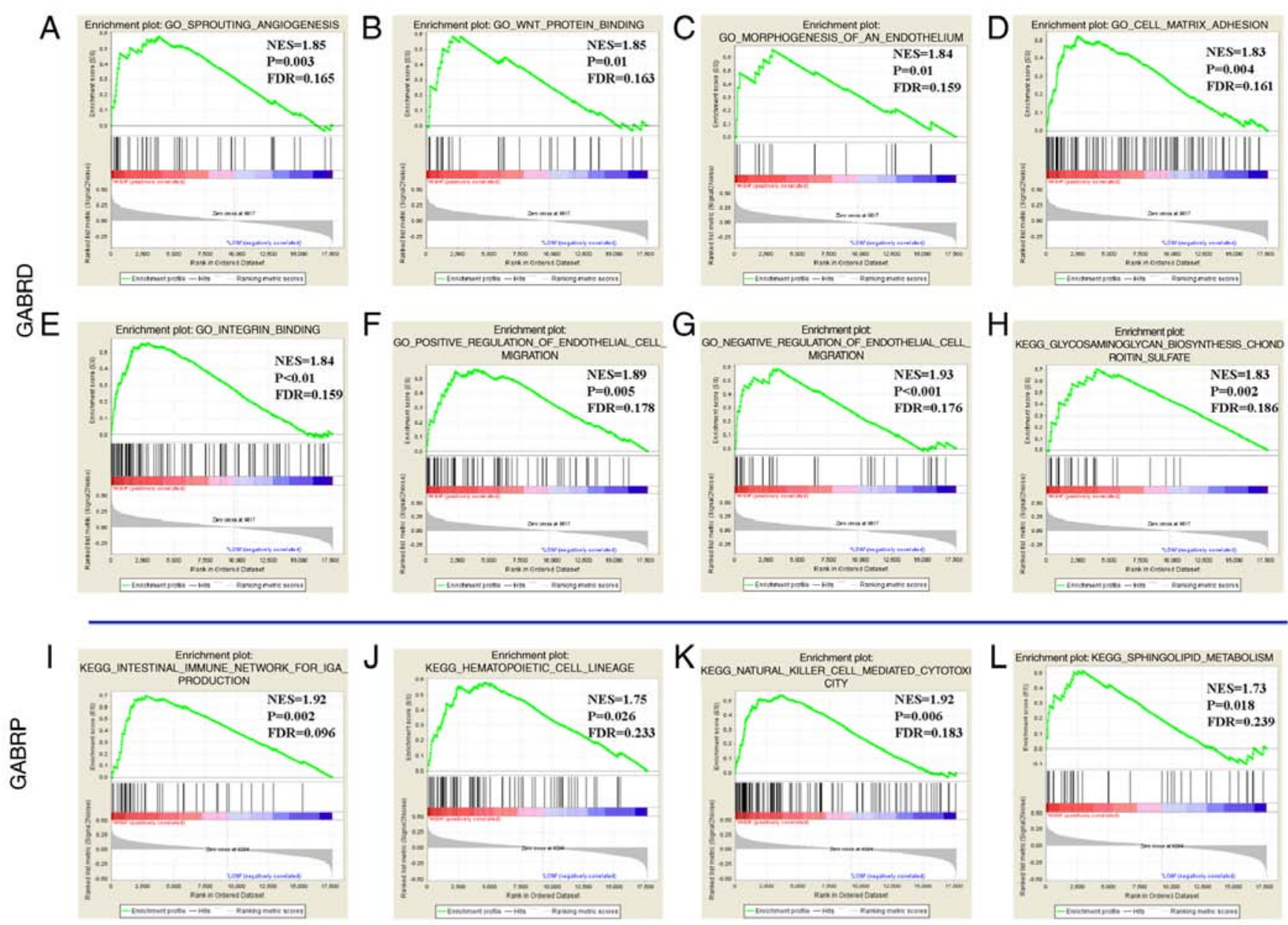

Figure 10. Gene Set Enrichment Analysis of GABRD and GABRP in patients with colon adenocarcinoma in The Cancer Genome Atlas dataset. (A-G) Results of c5 reference gene sets for high-GABRD expression groups. (H) Results of $\mathrm{c} 2$ reference gene sets for high-GABRD expression groups. (I-L) Results of c2 reference gene sets for high-GABRP expression groups. GABR, $\gamma$-aminobutyric acid type A receptor; NES, normalized enrichment score; FDR, false discovery rate.

function and fluidity 2)(53), Besim considered that signaling nodes in sphingolipid metabolism, such as sphingolipids, metabolic enzymes, and/or receptors, are new therapeutic targets for the development of new anticancer intervention strategies (54).

At present, few reports have been reported on GABRB1 in tumor field, our present study showed that $G A B R B 1$ was differentially expressed in tumor and adjacent normal tissues and that high expression levels of $G A B R B 1$ in patients with COAD was associated with a less favorable OS. Hence, GABRBI may also have potential as a prognosis biomarker of COAD.

In a previous study, GABRD was upregulated in patients with COAD and was not associated with proliferation (14), which is consistent with the results of the present study. Sarathi et al found GABRD was significantly monotonically upregulated across stages in hepatocellular carcinoma (55). In the present study, it was demonstrated that the expression of GABRD in COAD was significantly upregulated compared with normal tissues. Low expression levels of GABRD were associated with a more favorable prognosis and could be used as a biomarker for prognosis.

At present, it is known that $G A B R P$ serves a role in cancer development and progression. Menelaos et al found that GABRP gradually downregulated as tumors progressed, and it may serve as a prognostic marker for breast cancer (56). In contrast, Symmans et al (57) found increased expression of GABRP gene in undifferentiated cell type breast cancer and is significantly associated with shorter lifetime history of breastfeeding and with high-grade breast cancer in Hispanic women. Sung et al found that $G A B R P$ enhances aggressive phenotype of ovarian cancer cells (58). Jiang et al found that the expression of GABRP in pancreatic cancer tissues was significantly increased and associated with poor prognosis, contributing to tumor growth and metastasis (59). In our study, we found that the expression of $G A B R P$ in cancer tissues was higher than in adjacent normal tissues and high expression of GABRP are associated with poor prognosis of patients with COAD, which were consistent with the previous studies. It was also shown that OS was less favorable in patients with COAD with high expression levels of GABRP compared with patients with low expression levels of COAD.

Li et al (60) demonstrated that the overexpression of $G A B R Q$ was associated with the occurrence and development of HCC and might to become a molecular target for new diagnosis and treatment strategies for HCC. The multivariate COX proportional hazards model in the present study divided patients with COAD into groups based on high and low expression levels of $G A B R Q$ and showed that patients with high expression levels had a less favorable OS.

There were some limitations in the present study. First, the sample size was relatively small. Second, the clinical data were slightly inadequate, such as Event-free Survival (EFS) information, smoking, drinking history, tumor size and lymph node 
metastasis were not available from TCGA database. Therefore, it was not possible to perform a far-reaching survival analysis of $G A B A_{A}$ genes considering each potential prognostic variable of COAD in the multivariate Cox proportional hazards regression model. Third, although the association between the $G A B A_{A}$ gene family mRNA levels and COAD prognosis was investigated, the association between $G A B A_{A}$ family protein levels and COAD, $G A B A_{A}$ genes and GSEA still require further experimental research. Experiments like cell migration assays, detection of sulfuric acid related pathways at protein level and the functions of these genes in common cancer-related pathways, such as PI3K/AKT signaling pathway (61), JAK/STAT signaling pathway (62), should be conducted in future. However, despite these limitations, the present study further showed that the downregulated expression levels of GABRB1, GABRD, GABRP and $G A B R Q$ in COAD was associated with a more favorable prognosis and the potential mechanisms of GSEA associated with to $G A B R D$ and $G A B R P$ in the prognosis of COAD were studied. These results need to be verified with a larger sample size to confirm the role of the $G A B A_{A}$ family genes in the diagnosis and prognosis of COAD in the future.

Overall, the present study showed that the upregulated expression levels of GABRA2, GABRA3, GABRB2, GABRB3, GABRG2, GABRG3, GABRD and GABRE in COAD may have potential diagnostic value in COAD. In addition, the low expression levels of $G A B R B 1, G A B R D, G A B R P$ and $G A B R Q$ were associated with a more favorable prognosis of patients with COAD and could be used as a prognostic biomarker. Multivariate survival analysis, nomograms and joint survival analysis showed that the high expression of GABRB1, GABRD, $G A B R P$ and $G A B R Q$ were associated with poor prognosis of COAD. GSEA suggested that GABRD may impact cell adhesion, integrin binding, angiogenesis and so on; GABRP was associated with intestinal immune network for IGA production, hematopoietic cell lineage, and so on. However, the results of the present need to be confirmed by further research.

\section{Acknowledgements}

The authors thank the contributors of The Cancer Genome Atlas (portal.gdc.cancer.gov/) and proteinatlas.org for their contribution to share the colon adenocarcinoma dataset on open access.

\section{Funding}

The present study was supported by the Innovation Project of Guangxi Graduate Education (grant no. JGY2019052) and Self-financing Scientific Research Project of Guangxi Zhuang Autonomous Region Health Commission, China (grant no. Z20180959).

\section{Availability of data and materials}

The analyzed datasets generated during the study are available in The Cancer Genome Atlas repository (cancer.gov/tcga).

\section{Authors' contributions}

LY, MS and JG conceived and designed the study. XL, XW, $\mathrm{QH}, \mathrm{YG}, \mathrm{HX}$ and GR processed the data and performed the statistical analysis and they also generated and modified the figures. LY, LZ, XZ and FG wrote and revised the manuscript and helped to perform the analysis and interpretation of data. All authors read and approved the final manuscript.

\section{Ethics approval and consent to participate}

Not applicable.

\section{Patient consent for publication}

Not applicable.

\section{Competing interests}

The authors declare that they have no competing interests.

\section{References}

1. Fessler E and Medema JP: Colorectal cancer subtypes: Developmental origin and microenvironmental regulation. Trends Cancer 2: 505-518, 2016.

2. Takayama T, Ohi M, Hayashi T, Miyanishi K, Nobuoka A, Nakajima T, Satoh T, Takimoto R, Kato J, Sakamaki S and Niitsu Y: Analysis of K-ras, APC, and beta-catenin in aberrant crypt foci in sporadic adenoma, cancer, and familial adenomatous polyposis. Gastroenterology 121: 599-611, 2001.

3. IJspeert JE, Vermeulen L, Meijer GA and Dekker E: Serrated neoplasia-role in colorectal carcinogenesis and clinical implications. Nat Rev Gastroenterol Hepatol 12: 401-409, 2015.

4. Bray F, Ferlay J, Soerjomataram I, Siegel RL, Torre LA and Jemal A: Global cancer statistics 2018: GLOBOCAN estimates of incidence and mortality worldwide for 36 cancers in 185 countries. CA Cancer J Clin 68: 394-424, 2018.

5. Labianca R, Beretta GD, Kildani B, Milesi L, Merlin F, Mosconi S, Pessi MA, Prochilo T, Quadri A, Gatta G, et al: Colon cancer. Crit Rev Oncol Hematol 74: 106-133, 2010.

6. Fedirko V, Tramacere I, Bagnardi V, Rota M, Scotti L, Islami F, Negri E, Straif K, Romieu I, La Vecchia C, et al: Alcohol drinking and colorectal cancer risk: An overall and dose-response meta-analysis of published studies. Ann Oncol 22: 1958-1972, 2011.

7. Campbell PT, Cotterchio M, Dicks E, Parfrey P, Gallinger S and McLaughlin JR: Excess body weight and colorectal cancer risk in Canada: Associations in subgroups of clinically defined familial risk of cancer. Cancer Epidemiol Biomarkers Prev 16: 1735-1744, 2007.

8. Kuipers EJ, Grady WM, Lieberman D, Seufferlein T, Sung JJ, Boelens PG, van de Velde CJ and Watanabe T: Colorectal cancer. Nat Rev Dis Primers 1: 15065, 2015.

9. Darlison MG, Pahal I and Thode C: Consequences of the evolution of the GABA(A) receptor gene family. Cell Mol Neurobiol 25: 607-624, 2005 .

10. Mele M, Ferreira PG, Reverter F, DeLuca DS, Monlong J, Sammeth M, Young TR, Goldmann JM, Pervouchine DD, Sullivan TJ, et al: Human genomics. The human transcriptome across tissues and individuals. Science 348: 660-665, 2015.

11. Tian J, Lu Y, Zhang H, Chau CH, Dang HN and Kaufman DL: Gamma-aminobutyric acid inhibits T cell autoimmunity and the development of inflammatory responses in a mouse type 1 diabetes model. J Immunol 173: 5298-5304, 2004.

12. Macdonald RL and Olsen RW: GABAA receptor channels. Annu Rev Neurosci 17: 569-602, 1994.

13. Neelands TR and Macdonald RL: Incorporation of the pi subunit into functional gamma-aminobutyric Acid(A) receptors. Mol Pharmacol 56: 598-610, 1999.

14. Gross AM, Kreisberg JF and Ideker T: Analysis of matched tumor and normal profiles reveals common transcriptional and epigenetic signals shared across cancer types. PLoS One 10: e0142618, 2015.

15. Robinson MD, McCarthy DJ and Smyth GK: EdgeR: A bioconductor package for differential expression analysis of digital gene expression data. Bioinformatics 26: 139-140, 2010.

16. Mounir M, Lucchetta M, Silva TC, Olsen C, Bontempi G, Chen X, Noushmehr H, Colaprico A and Papaleo E: New functionalities in the TCGAbiolinks package for the study and integration of cancer data from GDC and GTEx. PLoS Comput Biol 15: e1006701, 2019. 
17. Huang da W, Sherman BT and Lempicki RA: Bioinformatics enrichment tools: Paths toward the comprehensive functional analysis of large gene lists. Nucleic Acids Res 37: 1-13, 2009.

18. Huang da W, Sherman BT and Lempicki RA: Systematic and integrative analysis of large gene lists using DAVID bioinformatics resources. Nat Protoc 4: 44-57, 2009.

19. Maere S, Heymans K and Kuiper M: BiNGO: A cytoscape plugin to assess overrepresentation of gene ontology categories in biological networks. Bioinformatics 21: 3448-3449, 2005.

20. Warde-Farley D, Donaldson SL, Comes O, Zuberi K, Badrawi R, Chao P, Franz M, Grouios C, Kazi F, Lopes CT, et al: The GeneMANIA prediction server: Biological network integration for gene prioritization and predicting gene function. Nucleic Acids Res 38 (Web Server Issue): W214-W220, 2010.

21. Montojo J, Zuberi K, Rodriguez H, Kazi F, Wright G, Donaldson SL, Morris Q and Bader GD: GeneMANIA cytoscape plugin: Fast gene function predictions on the desktop. Bioinformatics 26: 2927-2928, 2010.

22. Szklarczyk D, Franceschini A, Wyder S, Forslund K, Heller D, Huerta-Cepas J, Simonovic M, Roth A, Santos A, Tsafou KP, et al: STRING v10: Protein-protein interaction networks, integrated over the tree of life. Nucleic Acids Res 43 (Database Issue): D447-D452, 2015.

23. Shaul YD, Yuan B, Thiru P, Nutter-Upham A, McCallum S, Lanzkron C, Bell GW and Sabatini DM: MERAV: A tool for comparing gene expression across human tissues and cell types. Nucleic Acids Res 44 (D1): D560-D566, 2016.

24. Tang Z, Li C, Kang B, Gao G, Li C and Zhang Z: GEPIA: A web server for cancer and normal gene expression profiling and interactive analyses. Nucleic Acids Res 45 (W1): W98-W102, 2017

25. Liao X, Han C, Wang X, et al: Prognostic value of minichromosome maintenance mRNA expression in early-stage pancreatic ductal adenocarcinoma patients after pancreaticoduodenectomy. Cancer Manag Res 10: 3255-3271, 2018.

26. Tu HP, Ko AM, Chiang SL, Lee SS, Lai HM, Chung CM, Huang CM, Lee CH, Kuo TM, Hsieh MJ and Ko YC: Joint effects of alcohol consumption and ABCG2 Q141K on chronic tophaceous gout risk. J Rheumatol 41: 749-758, 2014.

27. Balachandran VP, Gonen M, Smith JJ and DeMatteo RP. Nomograms in oncology: More than meets the eye. Lancet Oncol 16: e173-e180, 2015

28. Subramanian A, Tamayo P, Mootha VK, Mukherjee S, Ebert BL, Gillette MA, Paulovich A, Pomeroy SL, Golub TR, Lander ES and Mesirov JP: Gene set enrichment analysis: A knowledge-based approach for interpreting genome-wide expression profiles. Proc Natl Acad Sci USA 102: 15545-15550, 2005.

29. Nayeem N, Green TP, Martin IL and Barnard EA: Quaternary structure of the native GABAA receptor determined by electron microscopic image analysis. J Neurochem 62: 815-818, 1994

30. Gumireddy K, Li A, Kossenkov AV, Sakurai M, Yan J, Li Y, Xu H, Wang J, Zhang PJ, Zhang L, et al: The mRNA-edited form of GABRA3 suppresses GABRA3-mediated Akt activation and breast cancer metastasis. Nat Commun 7: 10715, 2016.

31. Bautista W, Perez-Alvarez V, Burczynski F, Raouf A, Klonisch T and Minuk G: Membrane potential differences and GABAA receptor expression in hepatic tumor and non-tumor stem cells. Can J Physiol Pharmacol 92: 85-91, 2014.

32. Hackett CS, Quigley DA, Wong RA, Chen J, Cheng C, Song YK, Wei JS, Pawlikowska L, Bao Y, Goldenberg DD, et al: Expression quantitative trait loci and receptor pharmacology implicate Arg1 and the GABA-A receptor as therapeutic targets in neuroblastoma. Cell Rep 9: 1034-1046, 2014.

33. Chen ZA, Bao MY, Xu YF, Zha RP, Shi HB, Chen TY and He XH: Suppression of Human liver cancer cell migration and invasion via the GABAA receptor. Cancer Biol Med 9: 90-98, 2012.

34. Minuk GY, Zhang M, Gong Y, Minuk L, Dienes H, Pettigrew N, Kew M, Lipschitz J and Sun D: Decreased hepatocyte membrane potential differences and GABAa-beta3 expression in human hepatocellular carcinoma. Hepatology 45: 735-745, 2007.

35. Takehara A, Hosokawa M, Eguchi H, Ohigashi H, Ishikawa $\mathrm{O}$, Nakamura Y and Nakagawa $\mathrm{H}$ : Gamma-aminobutyric acid (GABA) stimulates pancreatic cancer growth through overexpressing GABAA receptor pi subunit. Cancer Res 67: 9704-9712, 2007.

36. Zhang YA, Liu HN, Zhu JM, Zhang DY, Shen XZ and Liu TT: RNA binding protein Noval promotes tumor growth in vivo and its potential mechanism as an oncogene may due to its interaction with GABA A Receptor-Y2. J Biomed Sci 23: 71, 2016.

37. Okegawa T, Pong RC, Li Y and Hsieh JT: The role of cell adhesion molecule in cancer progression and its application in cancer therapy. Acta Biochim Pol 51: 445-457, 2004.
38. Kawauchi T: Cell adhesion and its endocytic regulation in cell migration during neural development and cancer metastasis. Int J Mol Sci 13: 4564-4590, 2012.

39. Desgrosellier JS and Cheresh DA: Integrins in cancer: Biological implications and therapeutic opportunities. Nat Rev Cancer 10: 9-22, 2010.

40. Mouw JK, Yui Y, Damiano L, Bainer RO, Lakins JN, Acerbi I, Ou G, Wijekoon AC, Levental KR, Gilbert PM, et al: Tissue mechanics modulate microRNA-dependent PTEN expression to regulate malignant progression. Nat Med 20: 360-367, 2014

41. Kimura C, Hayashi M, Mizuno Y and Oike $M$ Endothelium-dependent epithelial-mesenchymal transition of tumor cells: Exclusive roles of transforming growth factor $\beta 1$ and 32. Biochim Biophys Acta 1830: 4470-4481, 2013.

42. Ghesquiere B, Wong BW, Kuchnio A and Carmeliet P Metabolism of stromal and immune cells in health and disease. Nature 511: 167-176, 2014.

43. Lee E, Pandey NB and Popel AS: Crosstalk between cancer cells and blood endothelial and lymphatic endothelial cells in tumour and organ microenvironment. Expert Rev Mol Med 17: e3, 2015.

44. Hanahan D and Weinberg RA: Hallmarks of cancer: The next generation. Cell 144: 646-674, 2011

45. Park SH, Kim BR, Lee JH, Park ST, Lee SH, Dong SM and Rho SB: GABARBP down-regulates HIF-1 $\alpha$ expression through the VEGFR-2 and PI3K/mTOR/4E-BP1 pathways. Cell Signal 26: 1506-1513, 2014

46. Ohsumi Y: Molecular dissection of autophagy: Two ubiquitin-like systems. Nat Rev Mol Cell Biol 2: 211-216, 2001

47. Mizushima N: The pleiotropic role of autophagy: From protein metabolism to bactericide. Cell Death Differ 12 (Suppl 2): S1535-S1541, 2005.

48. Zhu JH, Horbinski C, Guo F, Watkins S, Uchiyama Y and Chu CT Regulation of autophagy by extracellular signal-regulated protein kinases during 1-methyl-4-phenylpyridinium-induced cell death. Am J Pathol 170: 75-86, 2007.

49. Mizumoto S, Yamada S and Sugahara K: Molecular interactions between chondroitin-dermatan sulfate and growth factors/receptors/matrix proteins. Curr Opin Struct Biol 34: 35-42, 2015.

50. Willis CM and Kluppel M: Chondroitin sulfate-E is a negative regulator of a pro-tumorigenic Wnt/beta-catenin-Collagen 1 axis in breast cancer cells. PLoS One 9: e103966, 2014.

51. Ludvigsson JF, Neovius M, Ye W and Hammarstrom L: IgA deficiency and risk of cancer: A population-based matched cohort study. J Clin Immunol 35: 182-188, 2015.

52. Malmberg KJ, Carlsten M, Bjorklund A, Sohlberg E, Bryceson YT and Ljunggren HG: Natural killer cell-mediated immunosurveillance of human cancer. Semin Immunol 31: 20-29, 2017

53. Hannun YA and Obeid LM: Principles of bioactive lipid signalling: Lessons from sphingolipids. Nat Rev Mol Cell Biol 9: 139-150,2008.

54. Ogretmen B: Sphingolipid metabolism in cancer signalling and therapy. Nat Rev Cancer 18: 33-50, 2018.

55. Sarathi A and Palaniappan A: Novel significant stage-specific differentially expressed genes in hepatocellular carcinoma. BMC Cancer 19: 663, 2019.

56. Zafrakas M, Chorovicer M, Klaman I, Kristiansen G, Wild PJ, Heindrichs U, Knüchel R and Dahl E: Systematic characterisation of GABRP expression in sporadic breast cancer and normal breast tissue. Int J Cancer 118: 1453-1459, 2006.

57. Symmans WF, Fiterman DJ, Anderson SK, Ayers M, Rouzier R, Dunmire V, Stec J, Valero V, Sneige N, Albarracin C, et al: A single-gene biomarker identifies breast cancers associated with immature cell type and short duration of prior breastfeeding. Endocr Relat Cancer 12: 1059-1069, 2005

58. Sung HY, Yang SD, Ju W and Ahn JH: Aberrant epigenetic regulation of GABRP associates with aggressive phenotype of ovarian cancer. Exp Mol Med 49: e335, 2017.

59. Jiang SH, Zhu LL, Zhang M, Li RK, Yang Q, Yan JY, Zhang C, Yang JY, Dong FY, Dai M, et al: GABRP regulates chemokine signalling, macrophage recruitment and tumour progression in pancreatic cancer through tuning KCNN4-mediated $\mathrm{Ca}^{2+}$ signalling in a GABA-independent manner. Gut 68: 1994-2006, 2019.

60. Li YH, Liu Y, Li YD, Liu YH, Li F, Ju Q, Xie PL and Li GCl: GABA stimulates human hepatocellular carcinoma growth through overexpressed GABAA receptor theta subunit. World J Gastroenterol 18: 2704-2711, 2012.

61. Engelman JA: Targeting PI3K signalling in cancer: Opportunities, challenges and limitations. Nat Rev Cancer 9: 550-562, 2009.

62. Yu H, Lee H, Herrmann A, Buettner R and Jove R: Revisiting STAT3 signalling in cancer: New and unexpected biological functions. Nat Rev Cancer 14: 736-746, 2014

This work is licensed under a Creative Commons Attribution-NonCommercial-NoDerivatives 4.0 International (CC BY-NC-ND 4.0) License. 